\title{
Bcl11b/Ctip2 Controls the Differentiation of Vomeronasal Sensory Neurons in Mice
}

\author{
Takayuki Enomoto, ${ }^{1}$ Makoto Ohmoto, ${ }^{3}$ Tetsuo Iwata, ${ }^{1}$ Ayako Uno, ${ }^{1}$ Masato Saitou, ${ }^{1}$ Tatsuya Yamaguchi, ${ }^{1}$ \\ Ryo Kominami, ${ }^{4}$ Ichiro Matsumoto, ${ }^{3,5}$ and Junji Hirota ${ }^{1,2}$ \\ ${ }^{1}$ Department of Bioengineering, Graduate School of Bioscience and Bioengineering, and ${ }^{2}$ Center for Biological Resources and Informatics, Tokyo Institute \\ of Technology, Yokohama 226-8501, Japan, ${ }^{3}$ Department of Applied Biological Chemistry, Graduate School of Agricultural and Life Sciences, The University \\ of Tokyo, Tokyo 113-8657, Japan, ${ }^{4}$ Department of Molecular Genetics, Graduate School of Medical and Dental Sciences, Niigata University, Niigata \\ 951-8122, Japan, and ${ }^{5}$ Monell Chemical Senses Center, Philadelphia, Pennsylvania 19104-3308
}

The transcription factor Bcl11b/Ctip2 plays critical roles in the development of several systems and organs, including the immune system, CNS, skin, and teeth. Here, we show that Bcl11b/Ctip2 is highly expressed in the developing vomeronasal system in mice and is required for its proper development. Bcl1 1b/Ctip2 is expressed in postmitotic vomeronasal sensory neurons (VSNs) in the vomeronasal epithelium (VNE) as well as projection neurons and GABAergic interneurons in the accessory olfactory bulb (AOB). In the absence of $B c l 11 b$, these neurons are born in the correct number, but VSNs selectively die by apoptosis. The critical role of Bcl11b in vomeronasal system development is demonstrated by the abnormal phenotypes of $B c l 11 b$-deficient mice: disorganization of layer formation of the AOB, impaired axonal projections of VSNs, a significant reduction in the expression of vomeronasal receptor genes, and defective mature differentiation of VSNs. VSNs can be classified into two major types of neurons, vomeronasal 1 receptor $(\mathrm{V} 1 \mathrm{r}) / \mathrm{G} \alpha_{\mathrm{i} 2}$-positive and vomeronasal 2 receptor $(\mathrm{V} 2 \mathrm{r}) / \mathrm{G} \alpha_{\mathrm{o}}$-positive VSNs. We found that all $G \alpha_{i 2}$-positive cells coexpressed $G \alpha_{o}$ during embryogenesis. This coexpression is also observed in newly differentiated neurons in the adult VNE. Interestingly, loss of Bcl11b function resulted in an increased number of V1r/G $\alpha_{\mathrm{i} 2}$-type VSNs and a decreased number of V2r/G $\alpha_{\mathrm{o}}$-type VSNs, suggesting that Bcl11b regulates the fate choice between these two VSN types. These results indicate that Bcl11b/Ctip2 is an essential regulator of the differentiation and dichotomy of VSNs.

\section{Introduction}

Most terrestrial vertebrates possess a vomeronasal system, which detects pheromones to mediate social and reproductive behaviors (Keverne, 1999; Dulac and Torello, 2003; Halpern and Martínez-Marcos, 2003; Brennan and Zufall, 2006). In the mouse, pheromone signals are detected by the vomeronasal sensory neurons (VSNs), which generally express vomeronasal receptor (VR) genes that encode a putative seven-transmembrane domain protein (Dulac and Axel, 1995; Herrada and Dulac, 1997; Matsunami and Buck, 1997; Ryba and Tirindelli, 1997). There are two classic types of VSN, which are distinguished by the location of the cell body within the vomeronasal epithelium (VNE), the class of VR expressed, the type of G-protein subunit expressed,

Received March 10, 2011; revised May 26, 2011; accepted May 26, 2011.

Author contributions: J.H. designed research; T.E., M.O., T.I., A.U., M.S., T.Y., and J.H. performed research; R.K. and I.M. contributed unpublished reagents/analytic tools; T.E., M.O., and J.H. analyzed data; T.E. and J.H. wrote the paper.

This work was supported in part by the Ministry of Education, Culture, Sports, Science and Technology of Japan, Grant-in-Aid for Young Scientists (B) to M.0, Grant-in-Aid for Young Scientists (A) to I.M., and Grants-in-Aid for Scientific Research (C) to J.H.; by the National Institute on Deafness and Other Communication Disorders (Grant R03 DC011143) to I.M.; by the Japan Society for the Promotion of Science, Grant-in-Aid for Scientific Research on Innovative Areas to J.H.; and by grants from the Senri Life Science Foundation, the Inamori Foundation, and the Sumitomo Foundation to J.H. We thank Drs. P. Mombaerts and Y. Yoshihara for providing reagents, Dr. S. Fuss for instructions on the Dil experiment and critical review of the manuscript, and the members of the Hirota laboratory for their continuous support.

Correspondence should be addressed to Junji Hirota at the above address. E-mail: jhirota@bio.titech.ac.jp.

DOI:10.1523/JNEUROSCI.1245-11.2011

Copyright $\odot 2011$ the authors $\quad 0270-6474 / 11 / 3110159-15 \$ 15.00 / 0$ and their axonal target site in the accessory olfactory bulb (AOB). VSNs located apically in the VNE coexpress vomeronasal 1 receptor (V1r) genes along with $G \alpha_{i 2}$ and project their axons to the anterior half of the AOB. In contrast, basally located VSNs coexpress vomeronasal 2 receptor (V2r) genes along with $G \alpha_{o}$ and project their axons to the posterior half of the AOB (Berghard and Buck, 1996; Jia and Halpern, 1996). The cell bodies of V1r/G $\alpha_{\mathrm{i} 2}{ }^{-}$ positive and $\mathrm{V} 2 \mathrm{r} / \mathrm{G} \alpha_{\mathrm{o}}$-positive VSNs form nonoverlapping layers in the VNE. Although both types of VSNs are generated from Mash1-positive progenitor cells (Murray et al., 2003), the genetic mechanisms that regulate the differentiation and dichotomy of VSNs are not well understood; only a few critical transcription factors are known to regulate the development of VSNs (Murray et al., 2003; Ikeda et al., 2007; Duggan et al., 2008).

The Bcl11b gene (also known as Ctip2 or Rit1) encodes a C2H2 zinc finger transcription factor that is predominantly expressed in the immune system, CNS, and the embryonic olfactory system in mice (Avram et al., 2000; Wakabayashi et al., 2003a; Leid et al., 2004). Several studies of $B c l 11 b$-deficient $\left(B c l 11 b^{-/-}\right)$mice have demonstrated that $\mathrm{Bcl} 11 \mathrm{~b}$ is required for $\mathrm{T}$-cell development, the axonal projections of corticospinal motor neurons, the differentiation of medium spiny neurons of the striatum, skin morphogenesis, and odontogenesis (Wakabayashi et al., 2003b; Arlotta et al., 2005, 2008; Albu et al., 2007; Golonzhka et al., 2009a,b; Ikawa et al., 2010; Li et al., 2010a,b). In this study, we investigated the expression and function of $\mathrm{Bcl} 1 \mathrm{l} b$ in the developing vomeronasal system in vivo. We found that the expression of $B c l 11 b$ changed 
dynamically during the development of the VNE and AOB from embryogenesis to adulthood. The loss of function of Bcl11b resulted in severe abnormalities in the vomeronasal system, including the disorganization of layer formation in the $\mathrm{AOB}$, impaired axonal projection of VSNs, a severe reduction of $V R$ gene expression, defects in mature differentiation of VSNs, and distinct changes in the expression of several genes in VSNs. In addition, a deficiency of Bcl11b disturbs the balance between the two types of VSNs produced, suggesting that Bcl11b regulates the fate choice between these cell types, indicating that Bcl11b is a critical regulator during functional development of the vomeronasal system.

\section{Materials and Methods}

Mutant mice. Bcll $1 b^{-1-}$ and Mash $1^{-/-}$mice were generated as described previously(Guillemot et al., 1993; Wakabayashi et al., 2003b). Mutant and wild-type mice/embryos of either sex were used. For embryo staging, mid-day of the day of the vaginal plug was designated as embryonic day (E) 0.5 . The day of birth was designated postnatal day (P) 0 . All mouse studies were approved by the Institutional Animal Experiment Committee of the Tokyo Institute of Technology, and were performed in accordance with institutional and governmental guidelines.

In situ hybridization. Probes for Mash1, Ngn1, NeuroD, SCG10, GAP43, and $O M P$ were prepared as previously described (Hirota and Mombaerts, 2004). Probes for $G \alpha_{i 2}, G \alpha_{o}, V 1 r b 1, V 1 r d 16, V 1 r e 4, V 2 r a, V 2 r b$, and V2rc were provided by P. Mombaerts, Max-Planck Institute of Biophysics, Frankfort, Germany (Rodriguez et al., 2002; Ishii et al., 2003). Other probes were prepared from the cDNA of $B c l 11 b$ (nucleotides 1501-2560, GenBank accession number BC19503), Vill (nucleotides 356-2634, NCBI reference sequence NM_009509), Tcfap2e (nucleotides 916-1875, NCBI reference sequence NM_198960), Cart (nucleotides 96-788, NCBI reference sequence NM_001081493), Big2/Contactin4 (nucleotides 1189-2575, NCBI reference sequence NM_173004.3), Mef2b (nucleotides 500-1255, NCBI reference sequence NM_00445484), Panx3 (nucleotides 165-1598, NCBI reference sequence NM_172454), Meis2 (nucleotides 453-1354, NCBI reference sequence NM_001136072.2), and Olig1 (nucleotides 971-1919, NCBI reference sequence NM_016968.4). Single-color and two-color in situ hybridization (ISH) was performed according to a method described previously (Ishii et al., 2003, 2004). For two-color ISH, tyramide signal amplification (TSA)biotin, TSA-dinitrophenyl, and TSA-plus biotin systems (PerkinElmer) were used. The images were taken on an Olympus BX51 microscope with a DP71 digital CCD camera for bright-field images and a Leica SP or SPE confocal microscope for fluorescent images.

Immunohistochemistry. Immunohistochemistry (IHC) was performed according to a previously described method (Hirota et al., 2007). The following primary antibodies and dilutions were used: rabbit anti-Bcl11b (1:200) (Wakabayashi et al., 2003b); goat anti-Ki67 (1:1500 or 1:3000, catalog \#SC-7846, Santa Cruz Biotechnology); goat anti-OMP (1:5000, catalog \#544-10001, Wako); guinea pig anti-GABA (1:1000 or 1:10,000, catalog \#AB175, Millipore); rabbit anti-Tbx21 (1:5000, a gift from Y. Yoshihara, RIKEN, Wako, Japan) (Yoshihara et al., 2005); guinea pig anti-Tbx21 (1: 1000, a gift from Y. Yoshihara) (Yoshihara et al., 2005); mouse anti-NCAM (1:500, catalog \#C-9672, Sigma); mouse anti-synaptophysin (1:1000, catalog \#MAB5258, Millipore); rabbit anti-protocadherin 21 (1:1000, a gift from Dr. Yoshihara) (Kaneko-Goto et al., 2008); and rabbit anti-active caspase-3 (1: 500 or 1:5000, catalog \#C92-605, BD Pharmingen). The following appropriate secondary antibodies were used: the Alexa series (Invitrogen) and Cy3- and Cy5-conjugated goat anti-rabbit IgG (Jackson Immuno Research Laboratories); biotin-conjugated donkey anti-goat IgG (Rockland); biotinconjugated goat anti-guinea pig IgG and goat anti-rabbit IgG (Vector Laboratories) antibodies. For the immunostaining that used the anti-Ki67 or anti-active caspase- 3 antibodies, the sections were treated with the Vector Avidin/Biotin Blocking Kit (Vector Laboratories) and incubated with primary antibodies. Immunostaining was performed using the Vectastain Detection Kit (Vector Laboratories) in combination with the TSA-biotin system (PerkinElmer) to amplify signals. Anti-Ki67 immunoreactivity was detected using the DAB-chromogenic method with streptavidinHRP, and anti-active caspase-3 was detected with streptoavidinAlexa488 fluorescence. For immunostaining with the mouse anti-NCAM and anti-synaptophysin antibodies, the sections were pretreated with the Vector M.O.M. Immunodetection Kit (Vector Laboratories) before treatment with the primary antibody. For immunostaining of the nuclear protein, we performed antigen-retrieval pretreatment in $10 \mathrm{~mm}$ sodium citrate for $10 \mathrm{~min}$ or in HistoVT One (Nacalai Tesque) at $70^{\circ} \mathrm{C}$ for $15 \mathrm{~min}$ using a microwave oven.

Quantitative analyses. To quantify the number of DAPI-positive cells, $V R$-expressing cells, $G \alpha_{i 2}$ and $G \alpha_{o}$-expressing cells, proliferating cells, and apoptotic cells, every fifth or tenth coronal section $(10 \mu \mathrm{m}$ thickness), or every third coronal section ( $20 \mu \mathrm{m}$ thickness) throughout the vomeronasal organ (VNO) was collected for each staining experiment, and the number of positive cells was counted.

DiI tracing experiment. DiIC18(3) (DiI) crystal (Invitrogen) was heat melted to coat a glass capillary. After dissection of the heads of $B c l 11 b^{-1-}$ and wild-type mice, the nose tip was cut to expose the rostral vomeronasal organ, and the DiI-coated glass capillary was placed into the lumen of the VNO. Samples were incubated in PBS for $2 \mathrm{~h}$ at $37^{\circ} \mathrm{C}$, in $0.5 \%$ PFA/PBS overnight, and in $4 \%$ PFA/PBS for a month at room temperature to allow for the diffusion of DiI from the VSNs to the axonal termini. The DiI fluorescence of sagittally transected heads was imaged using an Olympus BX51 fluorescence microscope with a DP71-digital CCD camera.

Affymetrix microarrays. VNOs were obtained from $B c l 11 b^{-/-}$and wildtype mice at P0 and stored in RNAlater (Ambion). The total RNA of each preparation was extracted using the RNeasy Mini Kit (Qiagen). Biotinylated cRNA was synthesized using 10 ng of total RNA with the Two-Cycle Target Labeling and Control Reagents Kit (Affymetrix), fragmented, and hybridized to the DNA microarrays (Mouse Genome 4302.0 Array). To ensure reproducibility, microarray analyses were performed with RNA samples from six $B c l 11 b^{-/-}$and five wild-type mice. The microarray data were linearly normalized with the GAPDH (Probe ID: 1418625_s_at) signal of each preparation using GeneChip operating software (Affymetrix). The statistical significance of gene expression differences between $B c l 11 b^{-1-}$ and wild-type was analyzed using GeneSpring version 7.3 (Agilent Technologies), and differentially expressed genes with a false discovery rate $<0.25$ were extracted from the data for further experiments.

\section{Results}

$B c l 11 b$ is expressed in the developing vomeronasal epithelium The expression of Bcl11b/Ctip2 has been reported in the embryonic main olfactory epithelium (MOE) (Leid et al., 2004), but its expression in the VNE has not been studied. Because the MOE and the VNE are derived from the same olfactory placode, we assumed that $B c l 11 b$ would also be expressed in the VNE. Therefore, we examined the expression of $B c l 11 b$ in the developing VNE using ISH. The ISH studies revealed that $B c l 11 b$ was expressed in the VNE during the course of fetal development to adulthood, and its expression levels and patterns changed dynamically (Fig. $1 A$ ). The expression of $B c l 11 b$ was observed in the vomeronasal groove/VNE, and the MOE at the earliest time point examined, E11.5, which is shortly after the olfactory pits invaginate to develop the VNE and the MOE (Cuschieri and Bannister, 1975; Garrosa et al., 1998). The expression of Bcl11b increased gradually in level and in the number of cells during embryogenesis (Fig. $1 A$ ). From E16.5 to P0, a strong expression of Bcl1 $1 b$ was detected in the VSN layer but not in the sustentacular cell layer. Expression levels in individual cells were not uniform; rather, high-, low-, and nonexpressing cells were intermingled in the embryonic VNE. After birth, Bcl11b expression changed dynamically: $B c l 11 b$ expression gradually decreased and was restricted to the marginal region of the VNE (Fig. $1 A$, arrows), where neuronal progenitor/precursor cells and immature neurons localize. This result indicates that $B c l 11 b$ is expressed mainly in proliferating cells and/or immature neurons. Because proliferating cells and differentiating and differentiated neurons intermingle in the VNE during embryogenesis and for several days postnatally, the differential expression levels 
A

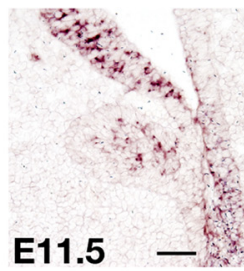

E12.5

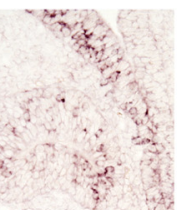

E14.5

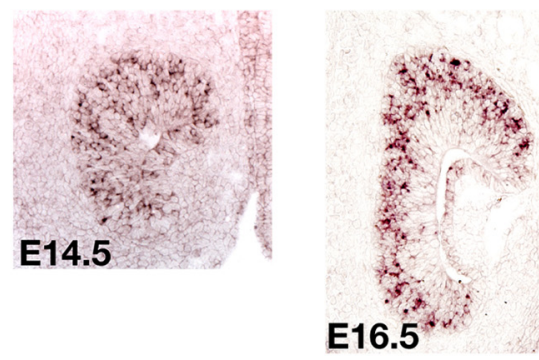

PO

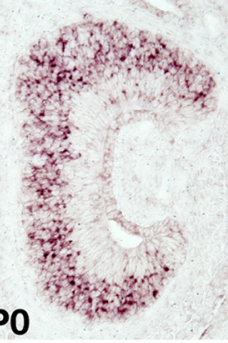

P60

P14

P14
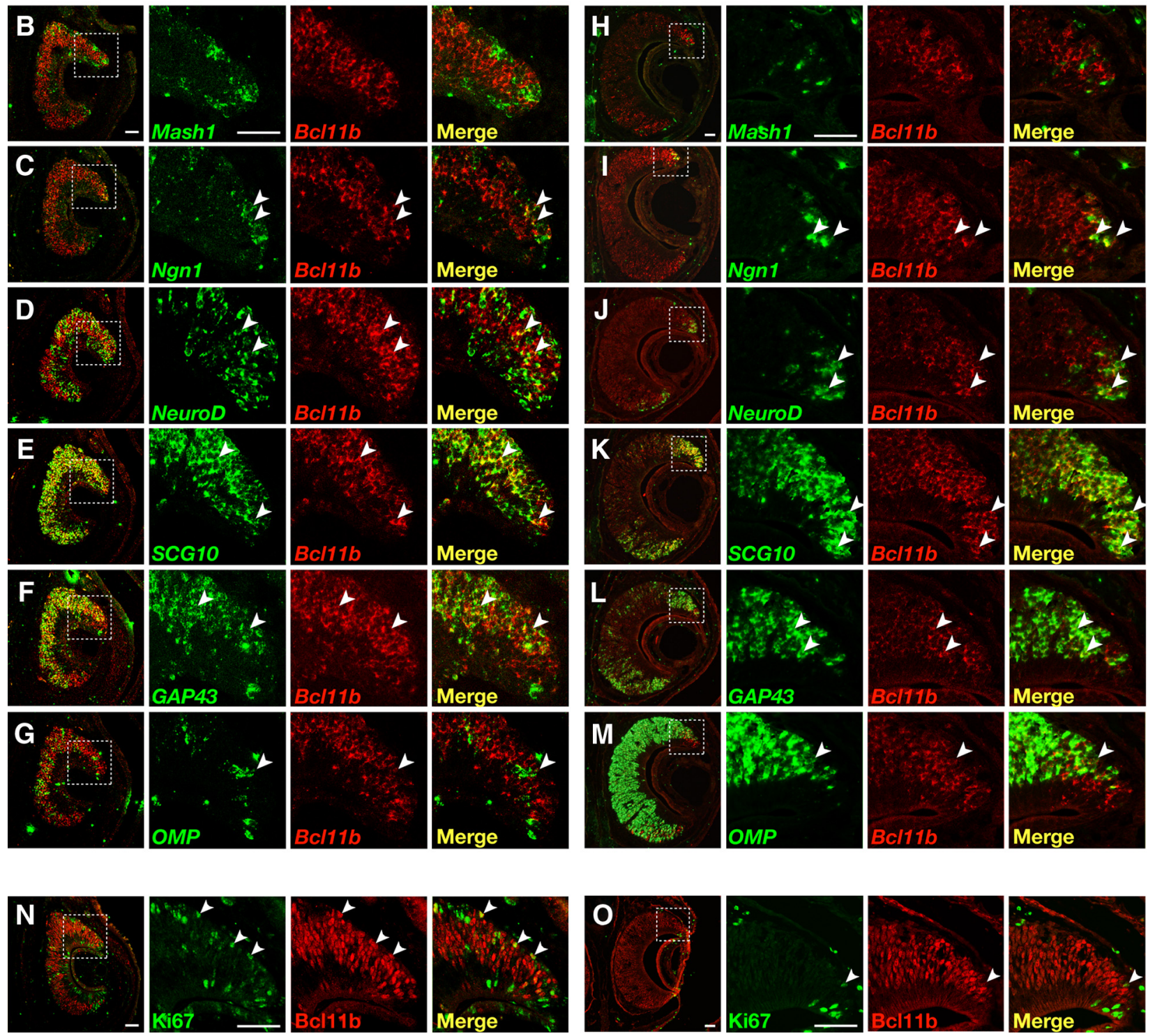

Figure 1. Expression of $B d 171 b$ in the developing mouse vomeronasal epithelium. $A$, In situ hybridization with RNA probes for $B d 171 b$ in coronal sections of the VNE at E11.5, E12.5, E14.5, E16.5, $\mathrm{P0}, \mathrm{P3}, \mathrm{P7}, \mathrm{P} 14$, and P60. The expression of $B C / 11 \mathrm{~b}$ gradually increased during embryogenesis. After birth, the expression of $B c / 11 \mathrm{~b}$ gradually decreased and was restricted (Figure legend continues.) 
of $B c l 11 b$ in individual cells in the embryonic VNE likely represent a differentiation-dependent expression of $B c l 11 b$.

To characterize Bcl11b-expressing cells, we performed twocolor ISH using riboprobes for specific molecular markers at $\mathrm{P} 0$ and P14 (Fig. $1 B-M$ ). In the MOE, Mash1 (a marker for neuronal progenitors), Ngn1 (neuronal precursors), NeuroD (differentiating/post mitotic neurons), SCG10 (pan-neurons/immature neurons), GAP43 (immature neurons), and OMP (mature neurons) were used to stage the differentiation of olfactory sensory neurons (OSNs) (Cau et al., 1997, 2002). However, the developmental time course of expression of these marker genes has not been fully established in the VSN lineage. Murray et al. (2003) have shown that the expression of Mash1 and Ngn1 can be detected in progenitor/precursor cells in the VNE; have demonstrated that Mash1 was required for Ngn1 expression in the VNE and for the neuronal differentiation of VSNs; and have proposed that a developmental hierarchy of gene expression in VSN lineage is similar to that of OSN. Accordingly, we used the same set of markers that were established for OSNs to characterize Bcl11b-expressing cells in the VNE.

At P0, Mash1-expressing cells did not coexpress Bcl11b (Fig. $1 B$ ), and a few Ngn1-expressing cells coexpressed Bcl11b (Fig. 1C). NeuroD was expressed in the developing VNE, and some NeuroD-positive cells were Bcl1 1b-positive in the VSN layer (Fig. $1 D$ ). SCG10 is expressed in immature neurons after terminal differentiation in the VNE and in the MOE (Camoletto et al., 2001). Most SCG10-positive cells highly expressed Bcl11b (Fig. $1 E)$. The same tendency was observed with another immature neuron marker, GAP43 (Fig. $1 F$ ). Expression of OMP partially overlapped with $B c l 11 b$ expression (Fig. $1 G$ ). At P14, the same pattern of coexpression for Bcl11b and differentiation markers was observed as at P0 (Fig. $1 H-M$ ). Because at this developmental time point expression of marker genes for neuronal precursors and immature neurons shifted to the marginal regions of the VNE, the expression of $B c l 11 b$ also became gradually restricted to these regions (Fig. 1). The distribution of SCG10-expressing cells overlapped well with high- $B c l 11 b$-expressing cells in the marginal region of the VNE (Fig. $1 \mathrm{~K}$ ). In contrast, most of the fully matured $O M P$-expressing cells were located in the central region of the VNE, which was complementary to the location of high$B c l 11 b$-expressing cells (Fig. $1 \mathrm{M}$ ). These results indicated that $B c l 11 b$ is first expressed between the late neuronal precursor stage and the immature neuron stage, and is highly expressed in postmitotic immature neurons. However, its expression level is gradually downregulated during mature differentiation. Additionally, double-label fluorescent IHC against the proliferation marker Ki67 (Schlüter et al., 1993) and Bcl11b showed that a small population of Ki67-positive cells is also positive for Bcl11b at P0 (Fig.

\section{$\leftarrow$}

(Figure legend continued.) to the marginal regions of the VNE in adulthood (arrows). $\boldsymbol{B}-\boldsymbol{M}$, $B d 111 b$-expressing cells were characterized using two-color ISH with RNA probes for $B d 111 b$ (red) in combination with marker genes (green) in coronal sections of the VNE at PO $(\boldsymbol{B}-\boldsymbol{G})$ and P14 (H-M): Mash1 (neuronal progenitors) $(\boldsymbol{B}, \boldsymbol{H})$; Ngn1 (neuronal precursors) (C, I); NeuroD (differentiating/postmitotic neurons) (D, J); SCG10 (immature neurons/pan-neurons) $(\boldsymbol{E}, \boldsymbol{K})$; GAP43 (immature neurons) $(\boldsymbol{F}, \boldsymbol{L})$; and OMP (mature neurons) $(\boldsymbol{G}, \boldsymbol{M})$. BCl11b was not coexpressed with Mash1 $(\boldsymbol{B}, \boldsymbol{H})$ but was partially coexpressed with $\operatorname{Ngn} 1$ ( $\boldsymbol{C}, \boldsymbol{I}$, arrowheads) and NeuroD ( $\boldsymbol{D}, \boldsymbol{J}$, arrowheads). Most of the $B \boldsymbol{C}(11 b$-positive cells were colabeled with the immature marker genes, SCG10 and GAP43 (E, $\boldsymbol{K}, \boldsymbol{F}, \boldsymbol{L}$, arrowheads). Expression of OMP expression was partially overlapped with that of $B C 111 b(\mathbf{G}, \mathbf{M}$, arrowhead). $\boldsymbol{N}, \mathbf{O}$, Double-label fluorescent IHC using an anti-Ki67 antibody, a proliferation marker and an anti-Bcl11b antibody showed that a small population of Ki67-positive cells colabeled with the anti-Bcl11b antibody (arrowheads), but most Bd11b-positive cells were Ki67-negative at PO $(\boldsymbol{N})$ and P14 (0). Scale bars, $50 \mu \mathrm{m}$.
$1 N)$ and P14 (Fig. 1O). Yet, most Bcl11b-positive cells are Ki67 negative. These results supported the above observations.

Because a few Bcl11b-expressing cells coexpressed $\mathrm{Ngn1}$ and NeuroD, but not Mash1, Bcl11b is probably located downstream of Mash1 in the VSN lineage. To test this possibility, we analyzed the expression of Bcl11b in Mash1 $1^{-/-}$VNE at E18.5 and found that no Bcl11b expression was observed (Fig. 2A). Therefore, Bcl11b might function downstream of Mash1 in the VSN lineage. Mouse VSNs are subdivided into two predominant types of neurons, the apical $G \alpha_{i 2}$ and $V 1 r$-positive neurons and the basal $G \alpha_{o}$ and $V 2 r$-positive neurons. To examine the VSN type-dependent expression of $B c l 11 b$, we performed two-color ISH of $B c l 11 b$ with $G \alpha_{i 2}, V 1 r d 16$ and $G \alpha_{o}, V 2 r a$ at P0. ISH showed that $B c l 11 b$ was expressed in both types of VSNs (Fig. 2B).

\section{Expression of Bcl11b in the accessory olfactory bulb}

We next examined $B c l 11 b$ expression in the developing AOB, which is the target of the axonal projections of VSNs by ISH, because expression in the embryonic $\mathrm{OB}$ was previously reported (Leid et al., 2004). Bcl11b was strongly expressed in the mitral/ tufted cell layer (M/TCL) and the granule cell layer (GCL) of the AOB during embryogenesis, and the expression gradually decreased after birth (Fig. $3 A$ ). After P14, weak expression of Bcl1 1b in the $\mathrm{AOB}$ was detected in the glomerular layer (GL) in addition to the M/TCL and the GCL (Fig. $3 A$, arrowheads). Therefore, $B c l 11 b$ shows spatially and temporally restricted expression patterns in the AOB and VNE.

To further characterize $B c l 11 b$-expressing cells in the AOB, we performed double-label fluorescent IHC on sagittal sections using antibodies against cell-type-specific proteins. Tbx21 is specifically detected in the projection neurons, the mitral/tufted cells in the main olfactory bulb and the AOB (Faedo et al., 2002; Yoshihara et al., 2005). At P0, we observed anti-Tbx21 immunostaining in the M/TCL only in the anterior portion of the AOB, but not in the posterior portion (Fig. 3B). Tbx21-positive cells overlapped with Bcl11b immunoreactivity in the anterior $\mathrm{AOB}$ (Fig. $3 B, D)$. At P14, anti-Tbx21-labeled cells were detected in the $\mathrm{M} / \mathrm{TCL}$ of both the anterior and posterior portions of $\mathrm{AOB}$ and now colabeled with the anti-Bcl1 lb antibody (Fig. 3G,I), indicating that Bcl11b-positive cells in the M/TCL are mitral/tufted cells. To characterize the Bcll1b-positive cells in the GL and GCL, coimmunostaining with anti-GABA antibody was conducted because there are many GABAergic interneurons in the GL and the GCL of the AOB (Takami et al., 1992). In addition to the M/TCL, $\mathrm{Bcl} 11 \mathrm{~b}$ protein was detected in the GCL at $\mathrm{P0}$ and in both the GCL and the GL at P14. At P0, some Bcl11b-positive cells in the GCL overlapped with GABA-positive interneurons (Fig. 3C,E). At P14, the immunostaining signal of the anti-GABA antibody was weaker compared with P0. Some Bcl11b-positive cells were also GABA positive in the GCL (Fig. $3 H, J$ ) and in the GL (Fig. $3 H, K$ ), indicating that at least a subpopulation of Bcll1b-positive cells in the GCL and the GL are GABAergic interneurons.

Our coexpression analysis revealed that the expression of $B c l 11 b$ changed dynamically during the development of the vomeronasal system in a temporally and spatially restricted manner. The strong expression of $B c l 11 b$ during embryogenesis suggests that Bcl11b might play important roles in the development of the vomeronasal system in mice.

\section{Impaired axonal projection of VSNs to the AOB in Bcl11b-deficient mice}

To investigate whether Bcl11b has an irreplaceable role in development of the vomeronasal system, we analyzed $B c l 11 b^{-/-}$mice at P0 
A

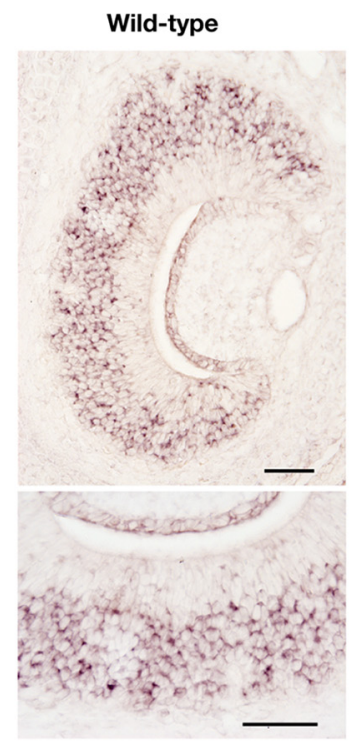

B
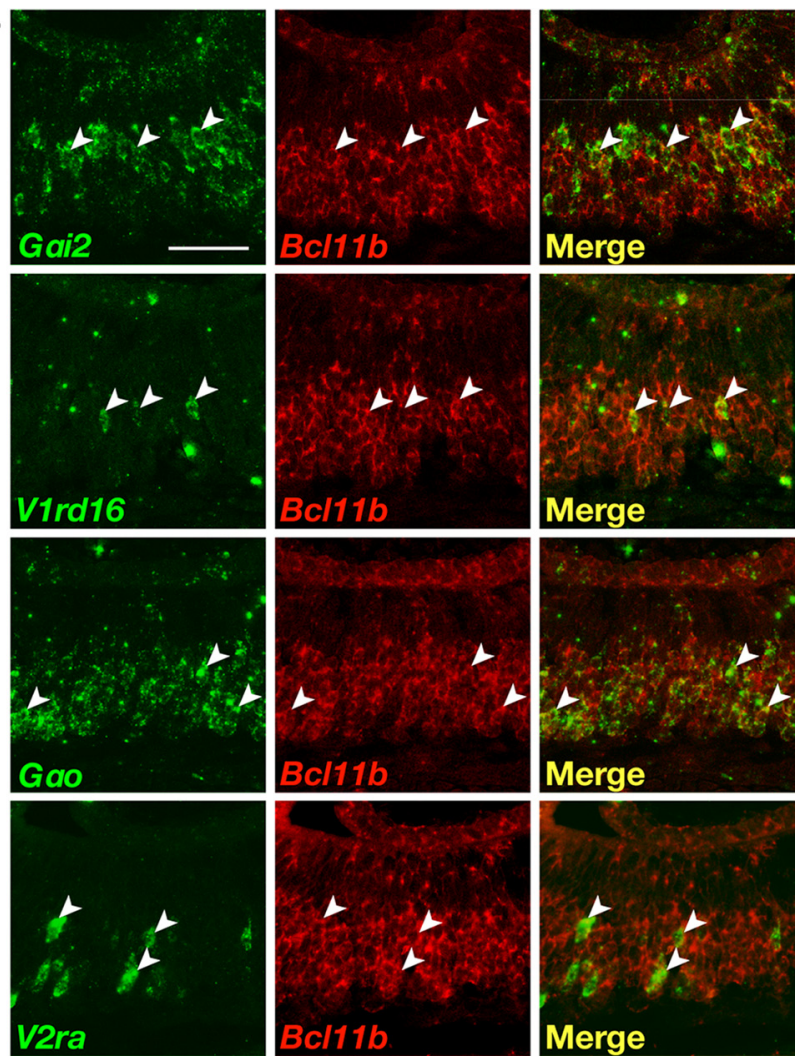

Figure 2. Bd11 b function downstream of Mash1 and in both two types of VSNs. $A$, Expression of $B d 171 b$ in $M a s h 1^{-1-}$ VNE was examined by ISH at E18.5. Expression of $B d 111 b$ was not observed in the Mash $1^{-/-}$VNE, indicating Bd11 b functions downstream of Mash1 in the VSN lineage. $\boldsymbol{B}$, To examine the VSN type-dependent expression of $B C 111 b$, we performed two-color ISH of $B C 111 b$ (red) with $G \alpha_{i 2}$, V1rd16, $G \alpha_{o}$, and V2ra (green). ISH showed that $B c 111 b$ was expressed in both types of VSNs (arrowheads). Arrowheads indicate typical colabeled VSNS. Scale bars, $50 \mu \mathrm{m}$.

and during embryonic stages because $B c l 11 b^{-1-}$ mice die within a day after birth. First, we analyzed $B c l 11 b^{-1-}$ mice by hematoxylineosin staining of coronal sections of the $\mathrm{VNE}$ and sagittal sections of the AOB. In the VNE, there were no morphological differences between $B c l 11 b^{-/-}$and wild-type mice throughout the developmental stages analyzed (Fig. 4A,B). Quantification of the size and cell numbers in the VNE were nearly identical between $B c l 11 b^{-/-}$and wild- type mice at P0 (size: $0.102 \pm 0.010 \mathrm{~mm}^{2} /$ section in $B c l 11 b^{-/-}$and $0.110 \pm 0.0060 \mathrm{~mm}^{2} /$ section in wild-type mice, $p=0.21$; cell numbers: $1879 \pm 118$ cells/section in $B c l 11 b^{-/-}$and $1955 \pm 148$ cells/ section in wild-type mice, $p=0.53 ; n=3$ ), which suggests that the VNE develops grossly normal in $\mathrm{Bcl1} 1 \mathrm{~b}^{-/-}$mice.

In contrast, differences in morphology between the $\mathrm{Bcll}_{1} \mathrm{~b}^{-/-}$ and wild-type animals gradually became obvious during development of the AOB (Fig. 4C). There was no difference in the morphology of the AOB between $\mathrm{Bcll}_{1} \mathrm{~b}^{-1-}$ and wild-type mice at E15.5, a time point at which the nerve layer is not yet observed. As the AOB is innervated by VSN axons, morphological differences become obvious. In wild-type mice, the AOB was clearly divided into five layers comprising the vomeronasal nerve layer (VNL)/GL, the external plexiform layer (EPL), the M/TCL, the internal plexiform layer/the lateral olfactory tract (IPL/LOT), and the GCL at P0, but the $B c l 11 b^{-/-}$AOB lacked the VNL/GL (Fig. $4 C)$. To confirm this observation, we performed IHC using antibodies against Tbx21 for mitral/tufted cells and against NCAM for VSN axons (Fig. 4D). In wild-type mice, as axons reach the AOB to form the VNL/GL, mitral/tufted cells shifted to deeper layers to form the M/TCL. In contrast, few VSN axons reached to the $B c l 11 b^{-/-} \mathrm{AOB}$; therefore, the mitral/tufted cells occupied a broad part of the AOB that corresponds to the VNL/GL, the EPL, and the M/TCL in wild-type mice (Fig. 4D). This impaired axonal projection was confirmed with double immunostaining for a presynaptic molecule of VSNs and a dendritic marker of mitral/ tufted cells using anti-synaptophysin and anti-pcdh21 antibodies, respectively. In wild-type mice, these immunostains were overlapped in the boundary between a synaptophysin-positive region and a pcdh21-region. In the $B c l 11 b^{-/-} \mathrm{AOB}$, however, less immunostaining for anti-synaptophysin antibody was observed, confirming that fewer axonal termini reached to the VNL/GL in the AOB (Fig. $4 E$ ).

Next, to visualize the axonal projections of VSNs to the AOB in their entirety, we performed DiI-tracing experiments by injecting a DiI-coated glass capillary into the lumen of the VNO. Figure $4 F$ shows the sagittally dissected $\mathrm{OB} / \mathrm{AOB}$ and nasal cavity and the top view of the AOB. In wild-type mice, axons were fasciculated to form several axon bundles that reached both the anterior and the posterior parts of the AOB. In $B c l 11 b^{-/-}$mice, DiI fluorescence was dimmer, and the axon bundles were narrower compared with the wild-type mice. Some axon bundles were directed predominantly to the anterior $\mathrm{AOB}$ rather than the posterior AOB. Other axon bundles were misdirected to somewhere in the OB. These results indicated that a Bcl11b deficiency caused a significant reduction in the axonal outgrowth from VSNs and impaired axonal projections to the AOB.

To further investigate whether there were any abnormalities caused by loss of Bcl11b activity in the AOB, we examined the production and survival of mitral/tufted cells, which are axonal target of VSNs. In the M/TCL, Tbx21-positive cells were observed in both $B c l 11 b^{-/-}$and wild-type mice at P0, and there was no obvious difference in the number of Tbx21-positive cells between $B c l 11 b^{-1-}$ and wild-type mice $(141 \pm 27.0$ cells/section in $B c l 11 b^{-/-}$and $137 \pm 14.4$ cells/section in wild-type mice, $p=$ $0.82 ; n=3)$, indicating that the primary postsynaptic neurons of VSNs were produced normally in $B c l 11 b^{-/-}$mice (Fig. 4D). Additionally, we observed few apoptotic cells in the AOB of both $B c l 11 b^{-/-}$and wild-type mice. These results indicated that the loss of Bcl11b function did not cause fatal defects in the production and survival of mitral/tufted cells in the AOB. 
A
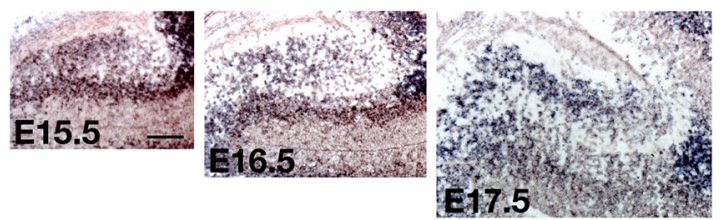

PO
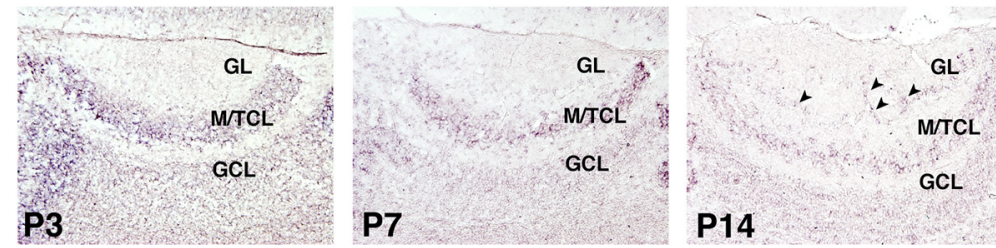

PO
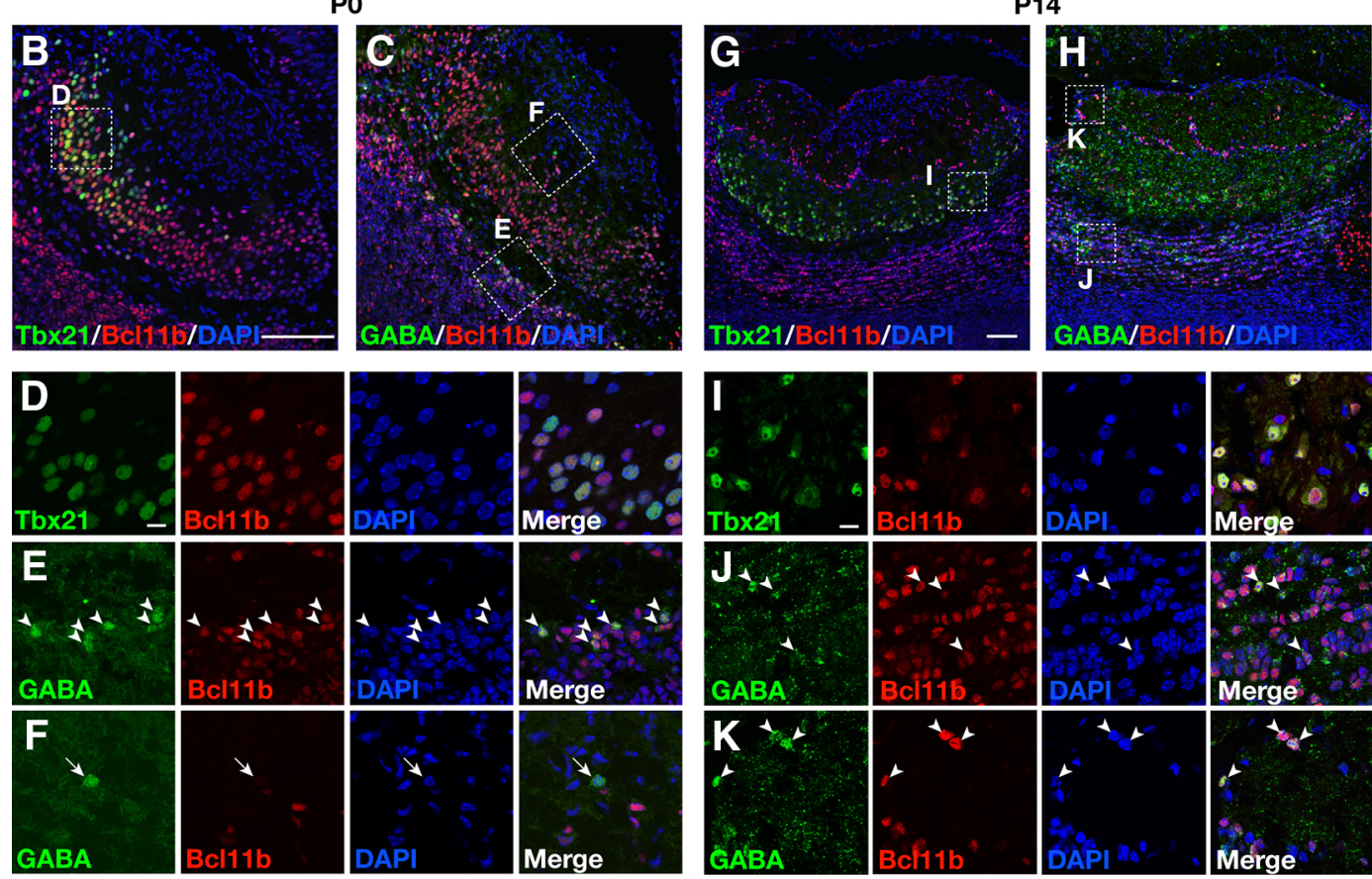

Figure 3. Expression of $B C / 11 b$ in the developing mouse accessory olfactory bulb. $A$, The expression of $B C / 11 b$ was examined using ISH in sagittal sections of wild-type AOB at E15.5, $\mathrm{E} 16.5, \mathrm{E} 17.5, \mathrm{P} 0, \mathrm{P} 3, \mathrm{P7}, \mathrm{P} 14$, and $\mathrm{P} 60 . B C 111 b$ was highly expressed in the $\mathrm{AOB}$ during embryogenesis. After birth, the expression of $B d 111 b$ decreased gradually. The expression of $B C l 11 b$ was observed in the M/TCL and the $\mathrm{GCL}$ at all developmental stages. The expression of $B C / 11 b$ in the $G L$ was detected after P14 (arrowheads). $\boldsymbol{B}-\boldsymbol{K}, \mathrm{BCl11b}$-positive cells were characterized using $\mathrm{IHC}$ with antibodies against specific marker proteins. Sagittal sections of the AOB at PO (B) and P14 (G) were immunostained with an anti-Tbx21 antibody (green: mitral cells) and an anti-Bcl11b antibody (red), and were counterstained with DAPI (blue: nucleus). $\boldsymbol{D}$ and $\boldsymbol{I}$ are higher-magnification images of the dotted box areas in $\boldsymbol{B}$ and $\boldsymbol{G}$, respectively. Bcl11b immunoreactivity was detected in all anti-Tbx21-labeled cells at PO and P14. $\mathbf{C}, \boldsymbol{H}$, Sagittal sections of the AOB at P0 $(\boldsymbol{C})$ and P14 $(\boldsymbol{H})$ were labeled with an anti-GABA antibody (green: GABAergic interneurons) and an anti-Bcl11b antibody (red), and were counterstained with DAPI (blue: nucleus). $\boldsymbol{E}$ and $\boldsymbol{J}$ as well as $\boldsymbol{F}$ and $\boldsymbol{K}$ are higher-magnification images of the dotted box areas of the $\mathrm{GCL}$ and the $\mathrm{GL}$ in $\mathbf{C}$ and $\boldsymbol{H}$, respectively. In the $\mathrm{GCL}, \mathrm{BCl} 11 \mathrm{~b}$ immunoreactivity was detected in some $\mathrm{GABAergic}$ neurons at both $\mathrm{P} 0$ and $\mathrm{P} 14(\boldsymbol{E}, \boldsymbol{J}$, arrowheads). In the $\mathrm{GL}$, Bcl11b immunoreactivity was colabeled with an anti-GABA antibody at P14 (K, arrowheads) but not at P0 (F, arrow). Scale bars: $\boldsymbol{A}-\boldsymbol{C}, \boldsymbol{G}, \boldsymbol{H}, 100 \mu \mathrm{m} ; \boldsymbol{D}-\boldsymbol{F}, \boldsymbol{I}-\boldsymbol{K}, 10 \mu \mathrm{m}$.

Incomplete development of VSNs in Bcl11b-deficient mice The impaired axonal outgrowth was indicative of the defective development of VSNs in $B c l 11 b^{-1-}$ mice. Therefore, we examined differentiation of VSNs in $\mathrm{Bcll} 1 \mathrm{~b}^{-/-}$mice at P0 by ISH using RNA probes for neuronal marker genes and expression levels of these genes with microarray analyses. The expression of Mash 1 in $B c l 11 b^{-/-}$was almost the same as in wild-type mice (Fig. $5 A$ ) (signal intensity of microarray analyses of probe set for Mash1, Probe ID, 1437086_at: $313 \pm 94.3$ in $\mathrm{Bcll1b}^{-/-}, n=6$, and $245 \pm$ 40.4 in wild-type, $n=5, p=0.17$ ), indicating that the generation of progenitor cells proceeds normally. Expression of $\mathrm{Ngn} 1$ and NeuroD were slightly but significantly increased in $B c l 11 b^{-1-}$ (Fig. 5A) (Ngn1, 1438551_at: $958 \pm 354$ in Bcl11 $b^{-/-}$and $494 \pm 86.5$ in wild-type mice, $p=0.020$; NeuroD, 1426412_at: $5476 \pm 803$ in $B c l 11 b^{-/-}$and $3541 \pm 775$ in wild-type mice, $\left.p=0.0030\right)$. There was no significant difference in the pan-neuronal/immature neuron marker gene, SCG10, between $B c l 11 b^{-/-}$and wild-type mice (Fig. 5A) (SCG10, 1423280_at: $6671 \pm 475$ in Bcl11 $b^{-/-}$and $7025 \pm 1276$ in wild-type, $p=0.053$ ), indicating that the terminal differentiation into neurons is not blocked. The expression of GAP43 was decreased (Fig. 5A) (GAP43, 1423537_at: $1753 \pm 192$ in Bcl11 b ${ }^{-1-}$ and $2774 \pm 556$ in wild-type mice, $p=0.0021, n=5)$. The expression of $O M P$ was severely reduced in $B c l 11 b^{-/-}$mice

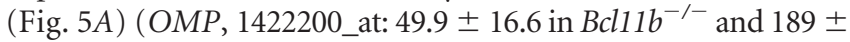
48.3 in wild-type mice, $p=0.00010$ ), which indicates that the differentiation of VSNs is arrested in postmitotic neurons in $B c l 11 b^{-/-}$ mice.

We extended our analyses to the fetal VNE. From E12.5 to E15.5 (Fig. $5 B, C$ ), when few $O M P$-positive mature neurons were observed in wild-type embryos, no obvious differences in the expression of Mash1, Ngn1, NeuroD, SCG10, and GAP43 were observed between $B c l 11 b^{-1-}$ and wild-type embryos. These re- 

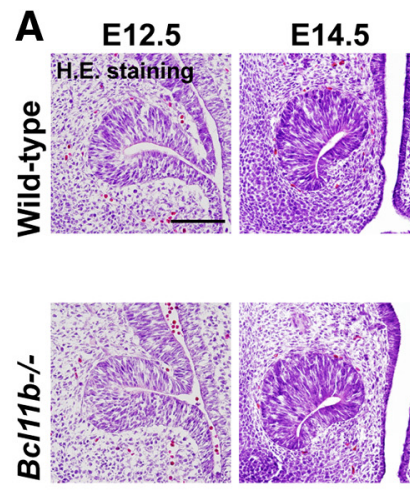

C

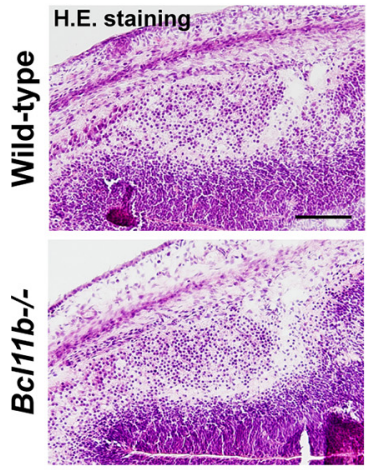

D

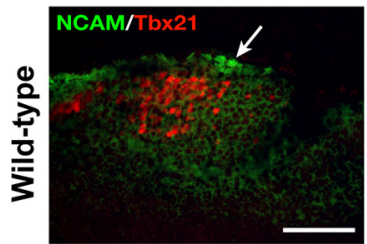

E15.5
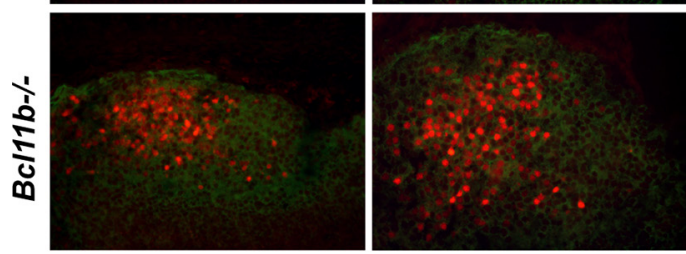

E
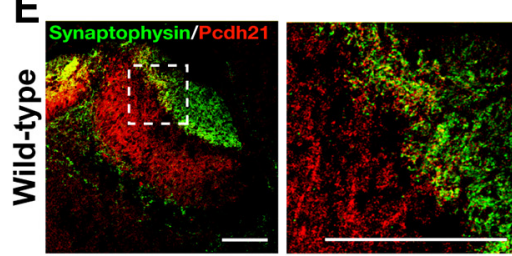

$\mathbf{F}$
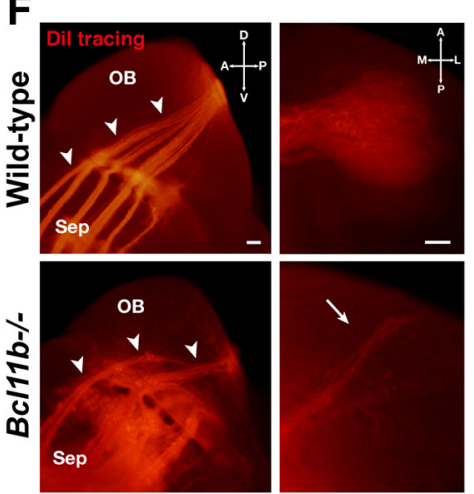

Figure 4. Impaired axonal projection of VSNs to the accessory olfactory bulb in $B C l 11 b^{-/-}$mice. $A$, Hematoxylin-eosin (H.E.) staining of coronal sections showed that the structure of the VNO appeared normal during the morphogenesis of the $B C l 11 b^{-/-}$ VNO at E12.5, E14.5, E16.5, and P0. B, The morphology of the VNO stained by DAPI was indistinguishable between $B C / 11 b^{-/-}$and wild-type mice at PO. C, H.E.-stained sagittal sections of the $B C / 11 b^{-/-}$and wild-type AOB at E15.5, E17.5, and P0. The layer organization of the $A O B$ gradually became obvious in the developing wild-type VNE but not in $B C / 11 b^{-/-}$mice due to the lack of the VNL/GL layer. D, Sagittal sections of $B C 111 b^{-/-}$and wild-type $A 0 B$ at E15.5, E17.5, and P0 were immunostained with an sults indicate that the production of progenitor/precursor cells and the terminal differentiation of VSNs proceed normally during early fetal development. After E16.5, when a severe reduction in $O M P$ expression became evident in $B c l 11 b^{-/-}$ compared with wild-type mice, Ngn1and NeuroD-expressing cells gradually increased in $\mathrm{Bcll1}^{-1-}$ mice. These results suggest that the slightly increased expression of Ngn1 and NeuroD after E16.5 are not due to a direct effect of the loss of function of Bcl11b. The increased expression is probably due to secondary effects that are caused by the reduction of mature VSNs, such as a reduction of feedback inhibition signals of neurogenesis from mature VSNs (Kawauchi et al., 2009).

Increased numbers of proliferative and apoptotic cells in Bcl11b-deficient mice The morphological normality of the VNE, the upregulation of Ngn1 and NeuroD expression, and the profound defects in mature differentiation of VSNs in $B c l 11 b^{-1-}$ mice at $\mathrm{P} 0$ suggested an alteration of the balance of proliferation and apoptosis in the VNE. To test this hypothesis, we quantified proliferative cells and apoptotic cells by immunostaining using anti-Ki67 and anti-active caspase- 3 antibodies, respectively (Fig. $6 A, B$ ). The number of proliferative cells was increased by $20 \%$ (303 \pm 11 cells/section in Bcl11 $b^{-/-}$and $253 \pm 14$ cells/section in wild-type mice, $p=0.0082$, $n=3$ ), and the number of apoptotic cells was increased by $820 \%$ (59 \pm 13 cells/section in $B c l 11 b^{-/-}$and $6.4 \pm 0.79$ cells/ section in wild-type mice, $p=0.0022, n=$ 3 ) at P0 (Fig. 6B). The normal morphology and cellular numbers in the VNE of

anti-NCAM antibody (green: axons) and an anti-Tbx21 antibody (red: mitral cells). No or extremely thin vomeronasal nerve layers (NCAM-positive) were observed in $B C / 11 b^{-/-}$ AOBs, but the Tbx21-positive mitral/tufted cells were widely distributed in the mutants. $\boldsymbol{E}$, Sagittal sections of the $A O B$ s of wild-type (top) and $B C / 11 b^{-/-}$(bottom) mice at PO were immunostained with an anti-synaptophysin antibody (green: presynapse) and an anti-Pcdh21 antibody (red: soma and dendrites of the mitral cells). High-magnification images of the dotted-box areas are shown in the right. $\boldsymbol{F}$, Whole-mount views of the vomeronasal axons labeled with Dil in wild-type (top) and BCl11 $b^{-1-}$ (bottom) mice at PO. The left panels show side views of the medial olfactory bulbs and nasal septa of sagittally transected mouse heads. The right panels show the top view of the caudal $O B$. Dil-positive fibers were observed along the nasal septum and extended to the $A O B$ (arrowheads) in both $B C 111 b^{-/-}$and wild-type mice. Axon fibers were decreased in number, and most of the axons did not reach the $A 0 B$ in $B C l 11 b^{-1-}$ mice (arrow). $A$, Anterior; $P$, posterior; $D$, dorsal; $V$, ventral; $M$, medial; $L$, lateral; Sep, septum of the main olfactory epithelium. Scale bars, $100 \mu \mathrm{m}$. 
A
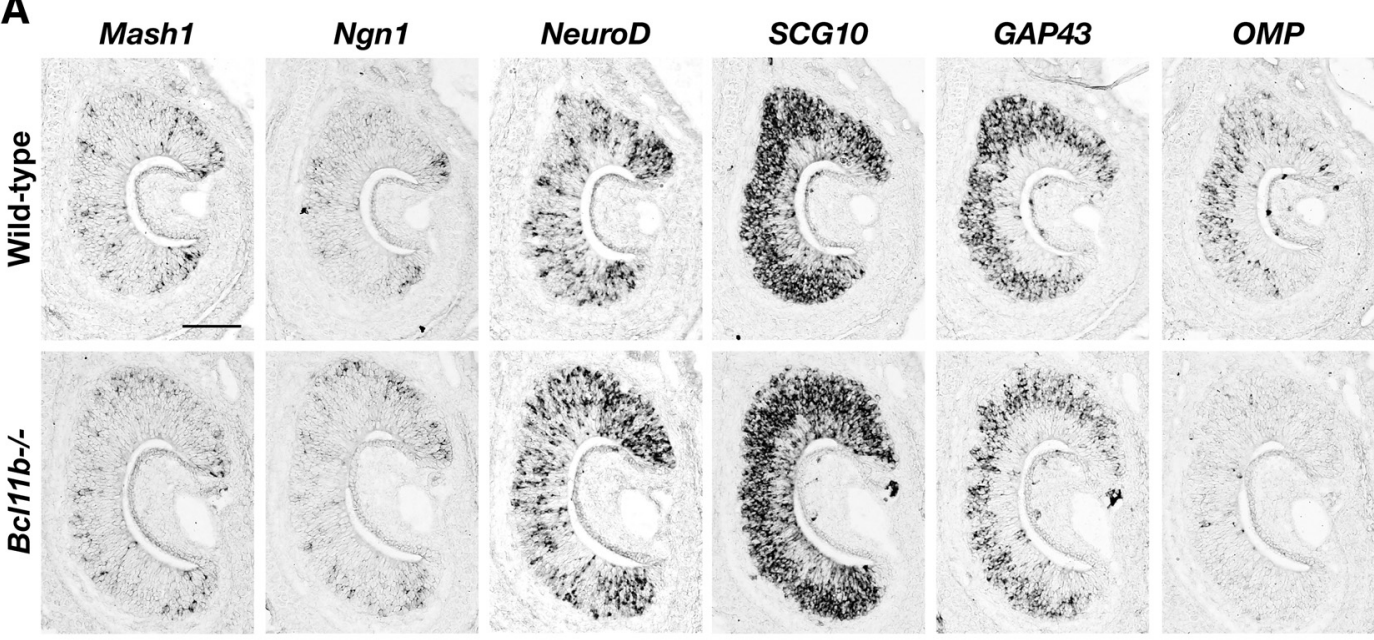

B
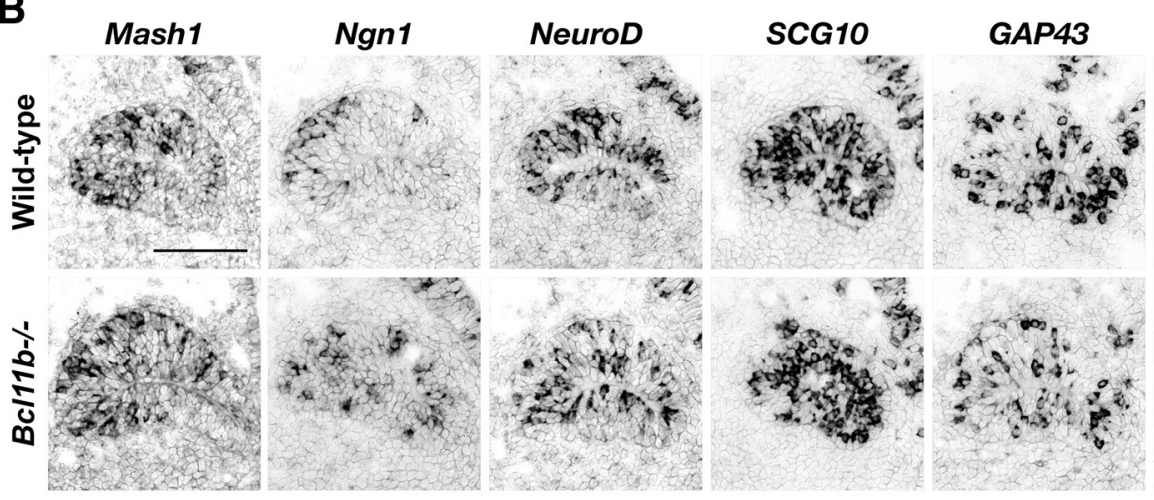

OMP
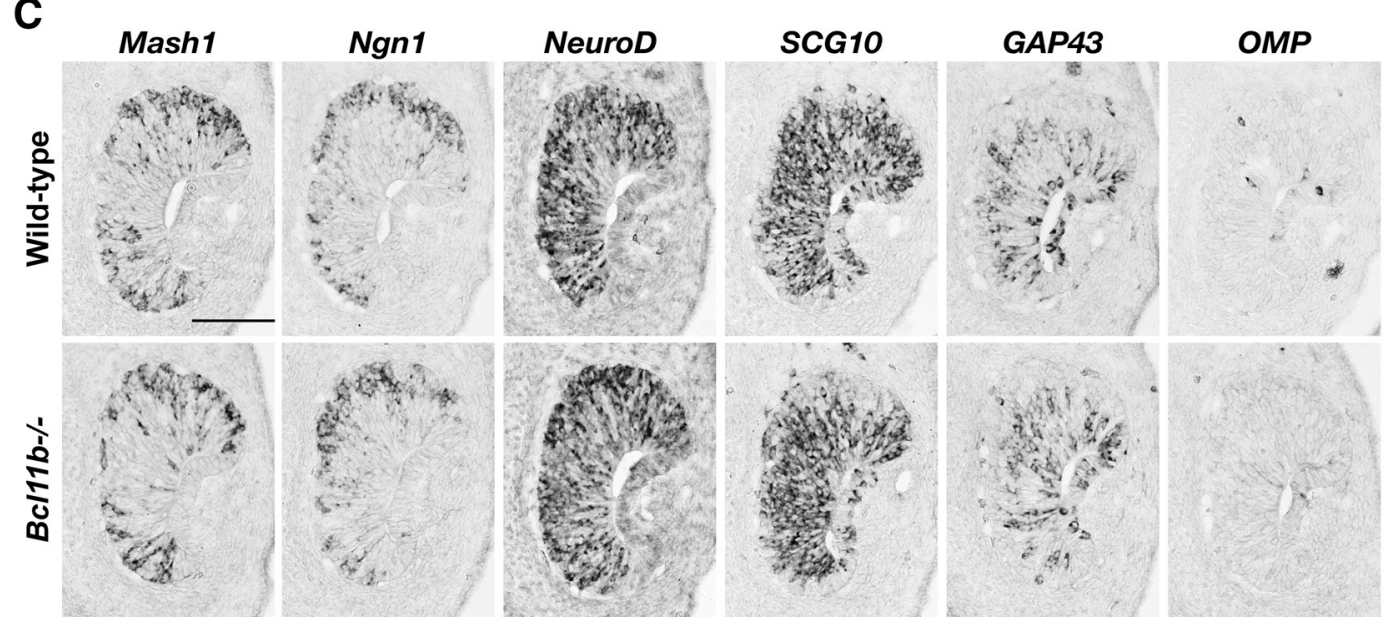

Figure 5. Incomplete development of VSNs in $B d 111 b^{-/-}$mice. $A$, The developmental defect of VSNs in the VNE of $B d 111 b^{-/-}$mice was characterized using ISH with the following RNA probes for neuronal markergenes:Mash1 (neuronal progenitors); Ngn1 (neuronal precursors); NeuroD (differentiating/postmitoticneurons); SCG10 (immatureneurons/pan-neurons); GAP43 (immatureneurons); and OMP(mature neurons) in coronal sections of wild-type and $B d 111 b^{-/-}$mice at PO. Similar expression patterns and levels of Mash1 were observed between $B d 111 b^{-/-}$and wild-type mice. Ngn 1 - and NeuroD-expressing cells were increased in $B d 111 b^{-1-}$ mice, but cells that expressed GAP43 and OMP were decreased. No obvious differences in the expression patterns SCG10 were observed between $B d 111 b^{-1-}$ and wild-type mice. $\boldsymbol{B}, \boldsymbol{C}$, The development and differentiation of VSNs werealso characterized using ISH during early fetal development atE12.5(B) and E15.5( $(\mathbf{C}$, a time point when OMP-positive matureneurons are rarely detected. No differences in the expression of Mash1, Ngn1, NeuroD, SCG10, or GAP43 were observed between Bc1116 ${ }^{-1-}$ and wild-type embryos. Scale bars, $100 \mu \mathrm{m}$.

$B c l 11 b^{-/-}$mice (Fig. $4 A, B$ ) could be due to an increased number of both apoptotic and proliferative cells (i.e., the net balance of dividing and dying cells might be almost the same as in the wild-type mice).

To define the step in VSN differentiation that apoptosis occurs in $B c l 11 b^{-1-}$ mice, we conducted double-immunostaining with anti-Ki67 and anti-active caspase-3 antibodies (Fig. 6C), and we performed ISH using GAP43 or OMP probes combined with the immunostaining of active caspase-3 (Fig. 6D,E). Active caspase-3-positive cells did not coimmunostain with the antiKi67 antibody, which indicates that proliferative cells hardly undergo apoptosis in $\mathrm{Bcl1} 1 \mathrm{~b}^{-/-}$and wild-type mice. Apoptotic cells were predominantly observed in GAP43-positive cells in $B c l 11 b^{-/-}$and wild-type mice (Fig. 6D). The number of GAP43positive apoptotic cells increased 10 -fold in the $B c l 11 b^{-/-} \mathrm{VNE}$ 

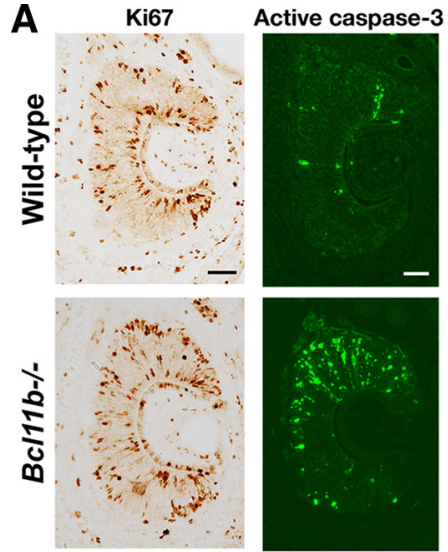

\section{C}
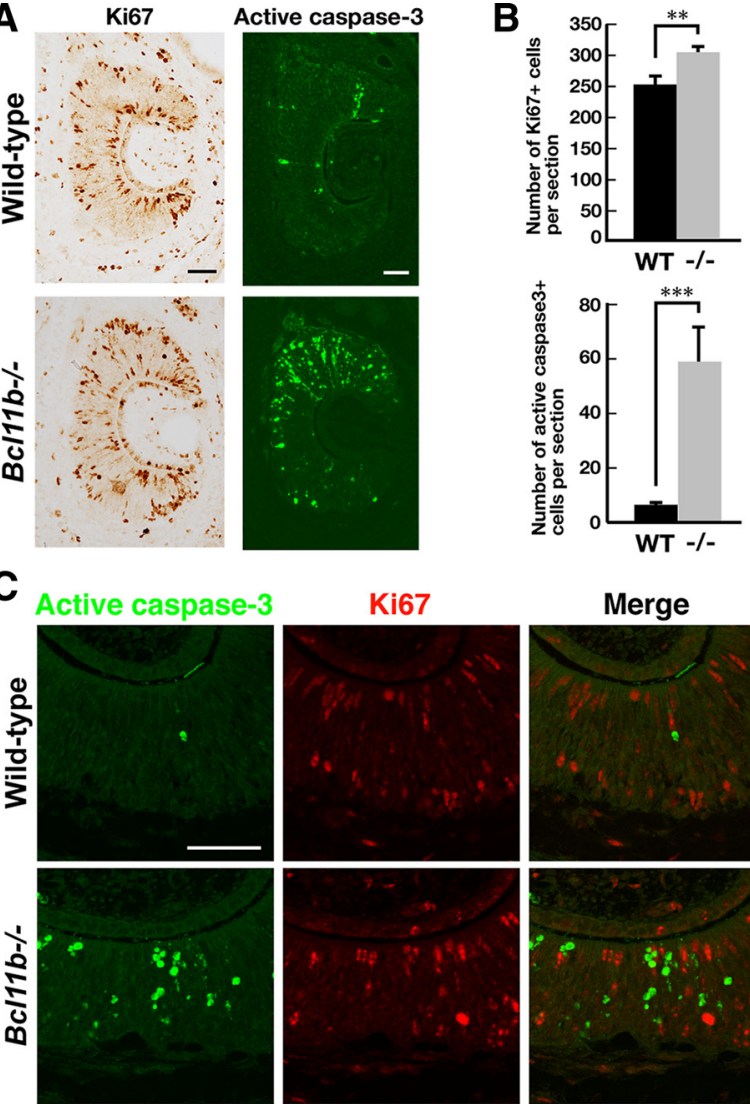

D Active caspase-3

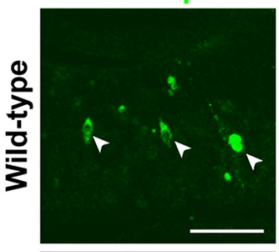

GAP43
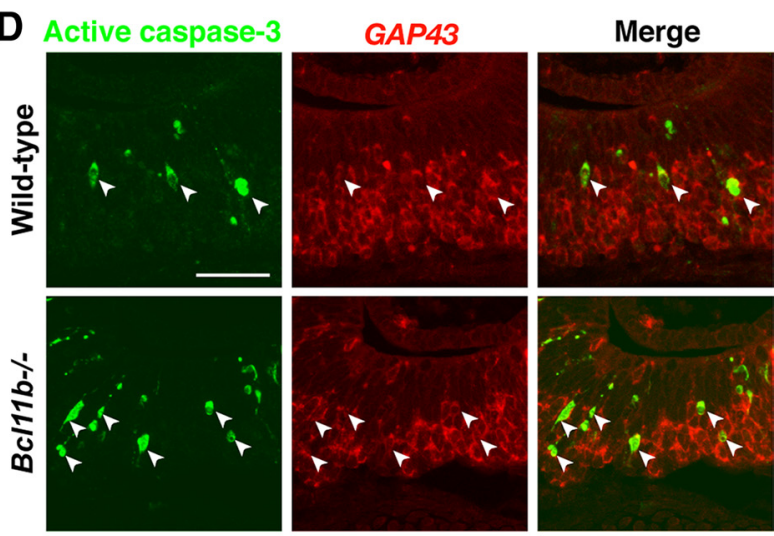

\section{E}
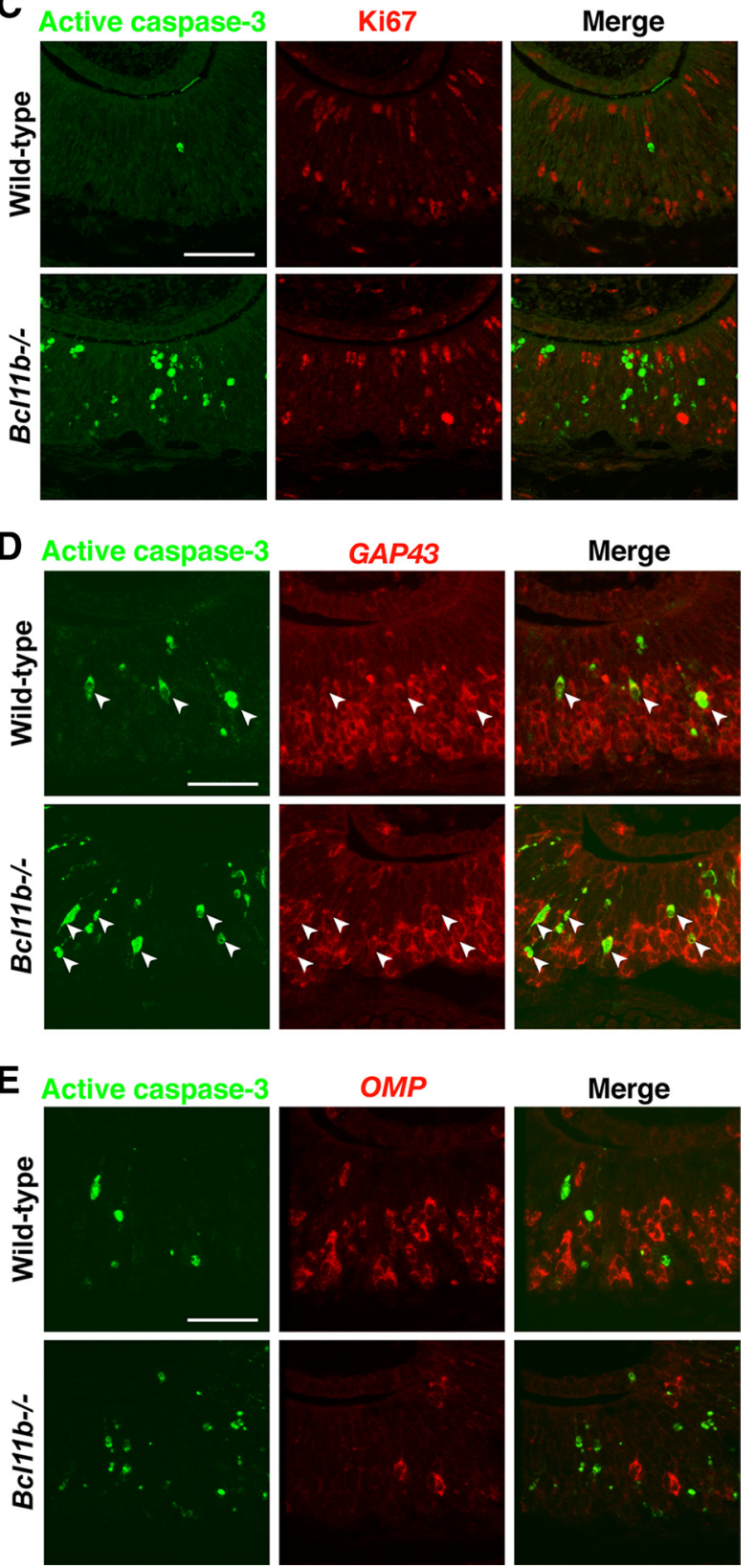

Figure 6. Increased proliferating cells and apoptotic cells in the VNE of $B C / 11 b^{-/-}$mice. $A$, Proliferative and apoptotic cells were immunostained with anti-Ki67 and anti-active caspase-3 antibodies, respectively. $\boldsymbol{B}$, The numbers of proliferative and apoptotic cells in the $B c 111 b^{-1-}$ and wild-type VNE were counted. The number of Ki67-positive proliferative cells was slightly $\left(29 \pm 6.1\right.$ cells/section in $B c l 11 b^{-/-}$and $2.6 \pm 0.73$ cells/section in wild-type mice, $p=0.0018, n=3$ ). In contrast, no significant difference in the number of $O M P$-positive apoptotic cells was observed between the $B c l 11 b^{-/-}$and wild-type VNE $(1.1 \pm 0.22$ cells/section in $B c l 11 b^{-1-}$ and $1.1 \pm 0.35$ cells/section in wildtype VNE, $p=0.87, n=3$ ) (Fig. $6 E$ ). Therefore, apoptosis occurs predominantly in the immature neuron stage in $\mathrm{Bcll}_{1} \mathrm{~b}^{-/-}$mice that results in a severe reduction in the number of mature VSNs.

\section{Identification of genes associated with the abnormal} differentiation of VSNs in Bcl11 b-deficient mice

Because Bcl11b functions as either a transcription activator or a repressor, depending on the cell type (Senawong et al., 2003; Topark-Ngarm et al., 2006; Marban et al., 2007; Cismasiu et al., 2008; Cherrier et al., 2009), a null mutation should result in a decrease or an increase in the expression of target and downstream genes. To identify genes whose expressions are associated with abnormalities in VSNs in Bcl11b-deficient mice, we directly compared gene expression profiles between the $\mathrm{Bcll}_{1} \mathrm{~b}^{-/-}$and wild-type VNOs at P0 using DNA microarray analysis and selected the most significantly downregulated or upregulated genes for further analysis. Because there were multiple cell types that were included in the dissected VNOs, we verified the microarray data using ISH and excluded those genes that were expressed in areas other than the VNE. We first found that the expression of both V1rs and V2rs, as indicated by the microarray data, was significantly reduced in $B c l 11 b^{-1-}$ mice (Table 1 ), suggesting that the abnormal differentiation of VSNs might associate with a reduction in the expression of $V R$ genes. Additionally, we identified eight genes that were either specifically downregulated (Vill, Panx3, Cart, Big2/Contactin4, Mef2b, and Tcfap2e) (Table 1, Fig. $7 A$ ) or upregulated (Meis2 and Olig1) (Table 1, Fig. 7B) in the $\mathrm{VNE}$ of $\mathrm{Bcl11} \mathrm{b}^{-/-}$mice. Among the downregulated genes identified, ISH analyses revealed that Vil1, Panx3, Cart, Big2/Contactin4, and Mef $2 b$ were expressed in both the apical and basal VSN layer, but Tcfap2e was expressed only in the basal VSN layer in wild-type mice (Fig. 7A). Because Big2/Contactin4 functions as an axonal guidance molecule in the main olfactory system, a severe reduction in Big2/Contactin4 could be involved in the phenotype of defective axonal projection of VSNs in $\mathrm{Bcll}_{11 b^{-/-}}$mice. Of the upregulated genes, Meis2 was expressed in the apical VSN layer in wild-type mice. Its expression was significantly increased and was detected in both the apical and basal portions in the $B c l 11 b^{-1-}$ VNE (Fig. 7B). The transcription factor Olig1 was not expressed in wild-type mice. However, it was expressed in $B c l 11 b^{-1-}$ mice, which indicates that the expression of Olig1 in the VNE is allowed by the absence of Bcl11b function. Therefore,

increased in $B C l 11 b^{-/-}$mice, and the number of active caspase-3-positive cells was significantly increased in $B C 111 b^{-/-}$mice. The error bars in $B$ represent the SD of the mean $(n=3$, Welch's or Student's $t$ test, ${ }^{* *} p<0.01,{ }^{* * *} p<0.005$ ). C, Coronal sections of the VNE at PO were labeled with an anti-active caspase- 3 antibody (green) and an anti-Ki67 antibody (red) in $B C / 11 b^{-1-}$ and wild-type mice. No coimmunostaining of Ki67 and active caspase- 3 was observed. $\boldsymbol{D}, \boldsymbol{E}$, Combinations of IHC and ISH were performed to define the step of differentiation when apoptosis occurred in $B C 117 b^{-1-}$ mice. Apoptotic cells were stained using $\mathrm{IHC}$ with an anti-active caspase-3 antibody ( $\boldsymbol{D}, \boldsymbol{E}$, green), and immature neurons and mature neurons were detected using ISH with probes for GAP43 (D, red) and $\operatorname{OMP}(\boldsymbol{E}$, red). Double-labeled cells were observed only in immature neurons ( $\boldsymbol{D}$, arrowheads), which indicated that apoptosis predominantly occurred in the immature stages. Scale bars, $50 \mu \mathrm{m}$. 
Table 1. Summary of the microarray analyses of gene expression in the $B C l 11 b^{-/-}$VNE

\begin{tabular}{|c|c|c|c|c|c|c|}
\hline \multirow[b]{2}{*}{ Gene name } & \multirow[b]{2}{*}{ Accession number } & \multirow[b]{2}{*}{ Probe ID } & \multicolumn{2}{|c|}{ Signal intensity } & \multirow[b]{2}{*}{ Fold change (KO/WT) } & \multirow[b]{2}{*}{$p$ value } \\
\hline & & & KO & WT & & \\
\hline \multicolumn{7}{|l|}{ VR genes } \\
\hline V1ra1 & NM_011683 & 1450558_at & $1.33 \times 10$ & $7.22 \times 10$ & 0.18 & $3.3 \times 10^{-3}$ \\
\hline V1ra2 & NM_011684 & 1427675_at & $1.42 \times 10$ & $2.54 \times 10^{2}$ & 0.06 & $6.3 \times 10^{-3}$ \\
\hline V1ra4 & NM_053219 & 1450598_at & $3.15 \times 10$ & $2.63 \times 10^{2}$ & 0.12 & $3.0 \times 10^{-3}$ \\
\hline V1ra5 & NM_053220 & 1422368_at & $1.21 \times 10$ & $3.94 \times 10$ & 0.31 & $1.3 \times 10^{-4}$ \\
\hline V1ra6 & NM_053221 & 1422369_at & $1.17 \times 10$ & $3.88 \times 10$ & 0.30 & $2.4 \times 10^{-4}$ \\
\hline V1rb2 & NM_011911 & 1421778_at & 4.10 & $3.39 \times 10$ & 0.12 & $6.5 \times 10^{-5}$ \\
\hline V1rd3 & NM_030740 & 1450343_at & 9.70 & $3.33 \times 10$ & 0.29 & $2.1 \times 10^{-3}$ \\
\hline V1rd9 & NM_030735 & 1421764_at & 5.80 & $4.13 \times 10$ & 0.14 & $4.3 \times 10^{-3}$ \\
\hline V1rd7 & NM_030737 & 1421724_at & $3.29 \times 10$ & $3.20 \times 10$ & 0.10 & $3.2 \times 10^{-3}$ \\
\hline V1rd14 & NM_030736 & 1450315_at & $7.02 \times 10$ & $2.25 \times 10^{2}$ & 0.31 & $3.5 \times 10^{-4}$ \\
\hline V2r1b & NM_019917 & 1421719_at & $1.60 \times 10$ & $8.01 \times 10$ & 0.20 & $3.0 \times 10^{-3}$ \\
\hline$V 2 r 4$ & NM_009493 & 1450331_s_at & $2.79 \times 10$ & $9.27 \times 10^{2}$ & 0.03 & $4.0 \times 10^{-3}$ \\
\hline V2r10 & NM_009494 & 1450338_x_at & $1.13 \times 10$ & $3.25 \times 10^{2}$ & 0.03 & $3.7 \times 10^{-3}$ \\
\hline V $2 r 16$ & NM_009491 & 1421701_at & $2.98 \times 10$ & $2.16 \times 10^{2}$ & 0.14 & $1.2 \times 10^{-3}$ \\
\hline V2r13 & NM_011686 & 1427681_s_at & $1.61 \times 10$ & $5.70 \times 10^{2}$ & 0.03 & $5.4 \times 10^{-3}$ \\
\hline \multicolumn{7}{|c|}{ Genes with decreased expression } \\
\hline Vil & NM_009509 & 1448837_at & 9.4 & $2.46 \times 10^{2}$ & 0.04 & $6.3 \times 10^{-3}$ \\
\hline Panx3 & NM_172454 & 1456073_s_at & $1.90 \times 10^{2}$ & $8.59 \times 10^{2}$ & 0.22 & $1.2 \times 10^{-3}$ \\
\hline Cart & NM_001081493 & 1422825_at & $8.63 \times 10$ & $7.49 \times 10^{2}$ & 0.12 & $1.4 \times 10^{-2}$ \\
\hline Cntn4 & NM_001109749 & 1460321_at & $1.13 \times 10^{2}$ & $6.17 \times 10^{2}$ & 0.18 & $4.5 \times 10^{-3}$ \\
\hline$M e f 2 b$ & NM_001045484 & 1421541_a_at & $9.29 \times 10$ & $4.56 \times 10^{2}$ & 0.20 & $5.0 \times 10^{-5}$ \\
\hline Tcfapze & NM_198960 & 1435205_at & $8.94 \times 10$ & $1.85 \times 10^{3}$ & 0.05 & $1.1 \times 10^{-3}$ \\
\hline \multicolumn{7}{|c|}{ Genes with increased expression } \\
\hline Meis2 & NM_001136072 & 1457632_s_at & $6.48 \times 10^{3}$ & $4.83 \times 10^{3}$ & 1.34 & $4.7 \times 10^{-3}$ \\
\hline Olig1 & NM_016968 & 1416149_at & $4.80 \times 10^{2}$ & $1.35 \times 10^{2}$ & 3.55 & $4.6 \times 10^{-4}$ \\
\hline
\end{tabular}

To identify genes whose expression is associated with abnormalities in $B d 111 b^{-/-}$mice, gene expression profiles between the $B d 111 b^{-/-}(n=6)$ and wild-type $(n=5)$ VNOs at PO were compared using microarray analysis. Signal intensities were linearly normalized with the GAPDH (Probe ID: 1418625 __at) signal of each preparation. Expression of most of Vr genes significantly decreased in the BCl11 $b^{-/-}$VN0. In addition, we identified the above listed eight genes that were either specifically downregulated or upregulated genes of potentially high biological relevance.
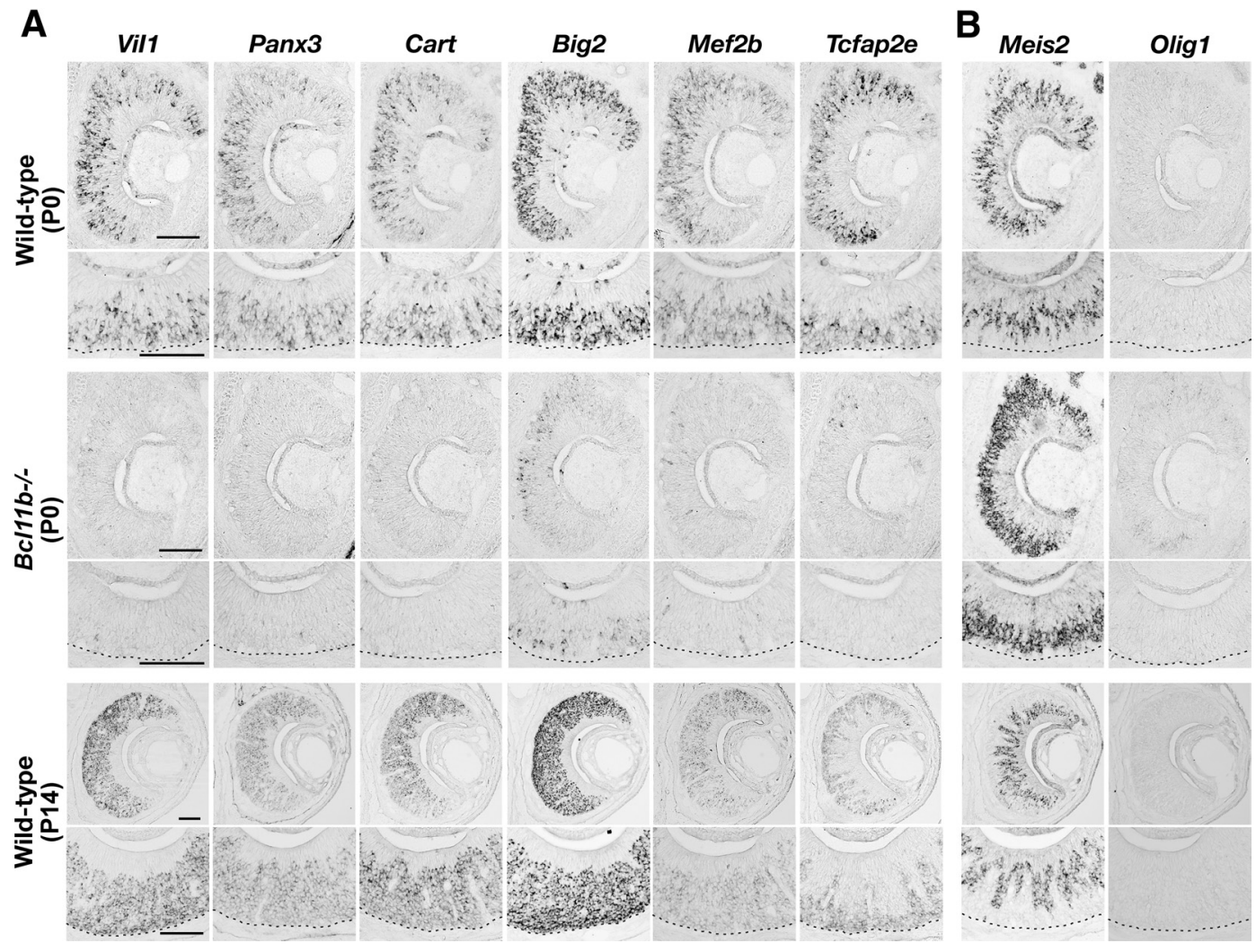

Figure 7. Changes in gene expression in the VNE of $B C 111 b^{-1-}$ mice. $A, B$, In situ hybridizations in coronal sections of wild-type (top panels) and Bcl11b ${ }^{-1-}$ (middle panels) VNE at P0, and wild-type VNE at P14 (bottom panels) showed genes with decreased expression $(\boldsymbol{A})$ or increased expression $(\boldsymbol{B})$ within the VNE of Bcl11b ${ }^{-1-}$ mice. Each set of panels consists of low-magnification (top) and high-magnification images (bottom). Dashed lines indicate the basal edge of the VNE. Tcfap2e and Meis2 are novel identifiers for the basal and apical VSNs, respectively. Scale bars, $100 \mu \mathrm{m}$. 

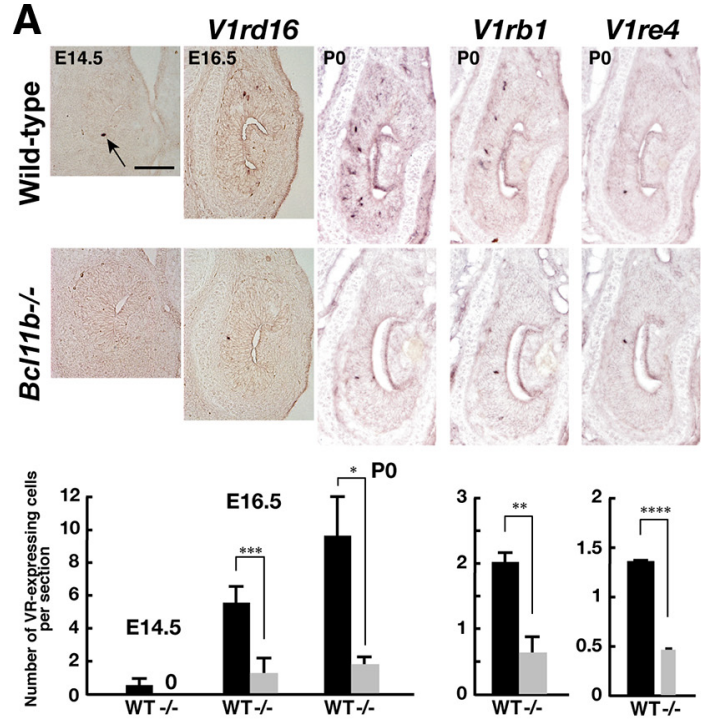

$\mathbf{C}_{A}$
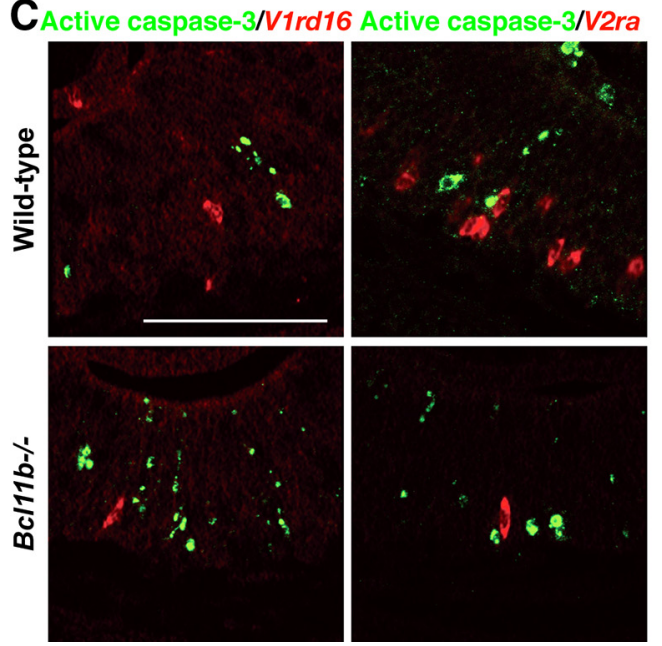
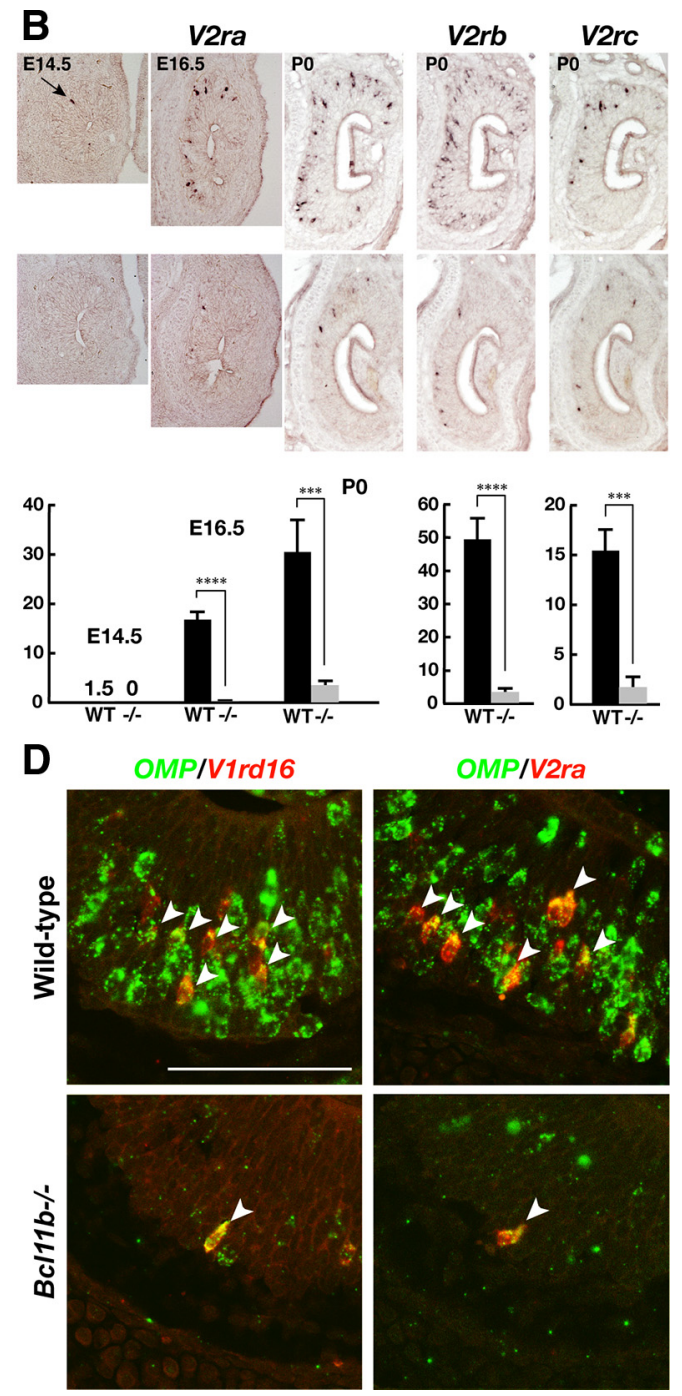

Figure 8. Severely reduced expression of the V1r and V2r genes in the VNE of $B d 11 b^{-1-}$ mice. $A, B$, The expression of the V1rs and V2rs genes was analyzed using ISH with the following probes: for V1rs: V1rd16 (at E14.5, E16.5, and P0), and V1rb1 and V1re4 (at P0); and for V2rs: V2ra (atE14.5, E16.5 and P0), and V2rb and V2rc (at P0). The lower graphs are quantifications of the number of VR-expressing cells in $B C l 11 b^{-/-}$(gray bar) and wild-type (black bar) mice. The expression of both V1rs and V2rs was significantly reduced in $B d 17 b^{-/-}$at all of the stages counted. The error bars in $A$ and $B$ represent the SD of the mean ( $n=3$; Welch's or Student's $t$ test, $\left.{ }^{*} p<0.05,{ }^{* *} p<0.01,{ }^{* * *} p<0.005,{ }^{* * * *} p<0.001\right)$. C, Apoptosis in VR-expressing VSNs was examined using a combination of ISH and IHC. None of the V1rd16-

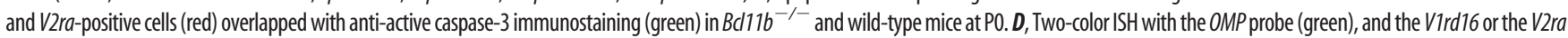
probe (red) in coronal sections of the VNE showed that VR-expressing VSNs differentiated to maturity in $B d 11 b^{-1-}$ mice (arrowheads indicate coexpression of OMP and VRs). Scale bars, $100 \mu \mathrm{m}$.

we identified eight candidate genes, in addition to the V1rs and $V 2 r s$, that may be located downstream of Bcl11b. Moreover, we identified Tcfap $2 e$ and Meis 2 as novel marker genes that are specifically expressed in the basal and apical VSNs, respectively. Interestingly, the expression of the novel basal marker Tcfap2e was downregulated, whereas the expression of the apical marker Meis2 was upregulated in $B c l 11 b^{-/-}$mice, which suggest that the deficiency of $B c l 11 b$ results in a decrease in basal VSNs and an increase in apical VSNs. These data demonstrate that the loss of Bcl11b function causes substantial changes in gene expression in the VNE and also provides a molecular foundation for the understanding of the function of Bcl11b in VSN differentiation.

\section{Severely reduced expression of $V 1 r$ and $V 2 r$ genes in} Bcl11b-deficient mice

To confirm the microarray data, we examined $V R$ gene expression in $\mathrm{Bcll}_{1} \mathrm{~b}^{-/-}$mice. We performed ISH using probes for the V1r family of V1rb1 (Vmn1r50), V1rd16 (Vmn1r180), and V1re4 (Vmn1r232); and for the $V 2 r$ family of $V 2 r a$ (Vmn2r8-17,
Vmn2r84-89, and Vmn2r121), V2rb (Vmn2r28-52), and V2rc (Vmn2r91-110). Quantification of the number of $V R$-expressing cells revealed that V1rs- and V2rs-expressing cells were severely decreased in $B c l 11 b^{-1-}$ mice at P0 [number of cells expressing $V 1 \mathrm{rb} 1$ (in cells/section) as follows: $0.63 \pm 0.23$ in $B c l 11 b^{-1-}$ vs $2.0 \pm 0.13$ in wild-type, $p=0.0024$; expressing V1rd16: $1.8 \pm$ 0.42 vs $9.6 \pm 2.3, p=0.025$; expressing V1re4: $0.47 \pm 0.06$ vs $1.3 \pm 0.03, p=0.00027$; expressing V2ra: $3.4 \pm 0.79$ vs $31 \pm 6.4$, $p=0.0019$; expressing $V 2 r b: 3.2 \pm 0.93$ vs $49 \pm 6.2, p=0.00023$; expressing $V 2 r c$ : $1.6 \pm 1.0$ vs $15 \pm 2.1, p=0.0023$ ] (Fig. $8 A, B$ ). The impact of $B c l 11 b$ deficiency on the reduction of V2rsexpressing cells (89-94\% reduction in $\mathrm{Bcl1}^{1} \mathrm{~b}^{-/-}$) is larger than on the reduction of V1rs-expressing cells (64-81\% reduction). The defective expression of $V R$ genes was also observed in $B c l 11 b^{-/-}$mice at E14.5 before the contact of vomeronasal axonal termini with the AOB (Fig. 8A,B) (V1rd16: none in $B c l 11 b^{-1-}$ and $0.57 \pm 0.41$ cells/section in wild-type mice; $V 2 r a$ : none in $B c l 11 b^{-/-}$and $1.5 \pm 0.66$ cells/section in wild-type mice), and at E16.5 (Fig. $8 A, B)\left(V 1 r d 16: 1.2 \pm 0.57\right.$ in Bcl11 $b^{-/-}$ 
and $5.6 \pm 0.84$ cells/section in wild-type mice, $p=0.0017 ; \mathrm{V} 2 \mathrm{ra}$ : $0.058 \pm 0.056$ in $B c l 11 b^{-/-}$and $17 \pm 1.6$ cells/section in wildtype mice, $p=0.000051$ ), suggesting that the decrease in the number of $V R$-expressing cells in $\mathrm{Bcll}_{1} \mathrm{~b}^{-/-}$mice is a cellautonomous effect of the loss of function of Bcl11b.

\section{$V R$-expressing cells in Bcl11b-deficient mice do not undergo apoptosis}

As described above, we found a profound defect of $V R$ gene expression in $B c l 11 b^{-/-}$mice, in addition to the increased apoptosis during the immature stage and incomplete mature differentiation of VSNs. To examine whether VR-expressing VSNs underwent apoptosis, we performed a combination of ISH and IHC using probes for V1rd16 and V2ra and an anti-active caspase- 3 antibody, respectively. In both $B c l 11 b^{-/-}$and wildtype mice, none of the V1rd16-positive and V2ra-positive cells overlapped with anti-active caspase-3 immunostaining, which indicates that neither V1rd16-expressing nor V2ra-expressing cells undergo apoptosis (Fig. 8C) (number of $\mathrm{V} 1 \mathrm{rd16}+$ cells counted: 169 in $\mathrm{Bcll}_{1} \mathrm{~b}^{-/-}$vs 881 in wild-type mice; number of V2ra + cells counted: 128 vs 2540 , respectively; $n=2)$. Therefore, increased apoptosis most likely occurs in non-receptorexpressing immature neurons in $B c l 11 b^{-/-}$mice. In addition, two-color ISH using probes for $V 1 r d 16$ and $V 2 r a$ vs OMP showed that most of the V1rd16- or V2ra-expressing cells were OMP positive (Fig. $8 D$ ), indicating that VRs-expressing VSNs have the potential to differentiate into mature VSNs.

\section{Bcl11b-mediated regulation of the dichotomy of VSNs}

During the quantification of the number of VRs-expressing neurons in the VNE of $B c l 11 b^{-/-}$mice, we found an abnormal distribution of these cells in $B c l 11 b^{-/-}$mice compared with wild-type mice. The V1rd16-expressing neurons were distributed broadly in both the apical and basal VSN layer in $B c l 11 b^{-/-}$mice (Fig. 8A), whereas the $V 2 \mathrm{ra}$-expressing cells were restricted more basally (Fig. $8 \mathrm{~B}$ ). Together with the microarray and ISH results showing the increased and broadening expression of the apical marker, Meis2, and the decrease in the basal marker, Tcfap $2 e$, in Bcl11 ${ }^{-1-}$ mice, it is conceivable that loss of Bcl11b function may differentially affect the differentiation pathway to the two VSN types (i.e., an increase in the apical VSN lineage and a decrease in the basal VSN lineage). To confirm this hypothesis, we investigated the expression of the G-protein $\alpha$ subunits, $G \alpha_{i 2}$ and $G \alpha$, which were specifically coexpressed in nonoverlapping layers in the adult VNE with V1rs and V2rs, respectively. The expression of $G \alpha_{i 2}$ was barely detectable at E14.5 in both the $B c l 11 b^{-/-}$and wild-type VNEs (Fig. 9A). At E16.5 and P0, the expression of $G \alpha_{i 2}$ was observed in both $B c l 11 b^{-1-}$ and wild-type mice, but its pattern was different. In wild-type mice, $G \alpha_{i 2}$ was expressed in the apical VSN layer. In $B c l 11 b^{-/-}$mice, however, $G \alpha_{i 2}$-expressing neurons increased in number and were broadly distributed in both the apical and basal VSN layers (Fig. 9A). On the other hand, the expression of $G \alpha_{o}$ was already detectable at E14.5 (Fig. $9 B$ ), suggesting that the onset of $G \alpha_{o}$ expression is earlier than that of $G \alpha_{i 2}$. At E16.5 and P0, $G \alpha_{o^{-}}$ expressing neurons were broadly distributed in both the $B c l 11 b^{-/-}$and wild-type VNEs, but the expression level of $G \alpha_{o}$ was obviously reduced in the Bcl11 $b^{-1-}$ VNE (Fig. 9B).

To further characterize $G \alpha_{i 2}$ and $G \alpha_{o}$-expressing cells, we performed two-color ISH for $G \alpha_{i 2}$ and $G \alpha_{o}$ (Fig. 9C). In wild-type mice at P0, we found that $G \alpha_{i 2}$-expressing VSNs coexpressed $G \alpha_{o}$, and these $G \alpha_{i 2} / G \alpha_{o}$ double-positive neurons were distributed in the apical VSN layer, whereas $G \alpha_{o}$ single-positive VSNs were located in the basal VSN layer. In contrast, this layer organization was not observed in $B c l 11 b^{-/-}$mice. In $B c l 11 b^{-/-}$mice, $G \alpha_{i 2}$-expressing VSNs also coexpressed $G \alpha_{o}$, and the number of $G \alpha_{i 2} / G \alpha_{o}$ double-positive neurons was significantly increased $(685 \pm 148$ neurons/section in $B c l 11 b^{-/-}$and $402 \pm 38.2$ neurons/section in wild-type mice, $p=$ $0.033 ; n=3)$, but $G \alpha_{o}$ single-positive neurons were severely decreased (26.9 \pm 6.05 neurons/section in $B c l 11 b^{-/-}$and $656 \pm 74.3$ neurons/section in wild-type mice, $p=0.0044 ; n=3$ ) (Fig. 9C). Most interestingly, two-color ISH revealed that $G \alpha_{i 2}$-positive cells were always $G \alpha_{o}$ positive in both $B c l 11 b^{-/-}$and wild-type mice at P0 (Fig. 9C). This coexpression was also observed in the marginal regions of the adult VNE, where proliferating cells and postmitotic immature neurons are located (Fig. 9D). Because there are no $G \alpha_{i 2}$ single-positive VSNs and there are already V1r-positive VSNs that exist at $\mathrm{P} 0$, it is possible to speculate that $G \alpha_{i 2} / G \alpha_{o}$ double-positive cells are the VSNs that are committed to the V1r-type lineage. To test this possibility, we examined coexpression of $V 1 r d 16$ with either $G \alpha_{o}$ or $G \alpha_{i 2}$ in wild-type mice at P0. Indeed, V1rd16-expressing VSNs coexpressed $G \alpha_{o}$ (63G $\alpha_{o}$-positive VSNs/63 V1rd16-positive VSNs) as well as $G \alpha_{i 2}$ (261 $G \alpha_{i 2}$-positive VSNs/263 V1rd16-positive VSNs) (Fig. 9E, left). In contrast, we found that V2ra-expressing VSNs only coexpressed $G \alpha_{o}$ (68 $G \alpha_{o}$-positive VSNs/68 V2rapositive VSNs), but not $G \alpha_{i 2}\left(0 G \alpha_{i 2}\right.$-positive VSN/242 V2rapositive VSNs) (Fig. 9E, right). These results indicate that $G \alpha_{i 2} / G \alpha_{o}$ double-positive VSNs commit to the V1r-VSN lineage, and V1r/G $\alpha_{\mathrm{i} 2}$ type of VSNs would be differentiated via the $G \alpha_{i 2} / G \alpha_{o}$ double-positive stage. In $B c l 11 b^{-/-}$mice, the number of cells in the VNE and the expression of the pan-neuronal/immature neuron marker, SCG10, were unaltered compared with wild-type mice (Figs. $4 B, 5$ ). These results suggest that the terminal differentiation into neurons proceeds normally, and almost the same number of VSNs is produced in $B c l 11 b^{-/-}$and wildtype mice. In $B c l 11 b^{-/-}$mice, the number of $G \alpha_{i 2}$-positive $\left(G \alpha_{i 2} /\right.$ $G \alpha_{o}$ double-positive) cells increased, whereas the number of $G \alpha_{o}$ single-positive cells decreased compared with that of wild-type mice, which demonstrates the opposite impact of Bcl11b deficiency on the generation of the two types of VSNs. Therefore, these results indicate that Bcl1 lb functions in the step that determined the fate of VSN types.

\section{Discussion}

Here, we report that $B c l 11 b$ is expressed in the vomeronasal system of mice and is required for its proper development. We demonstrate that the loss of Bcll1b results in impaired axonal projections of VSNs, a significant reduction in the expression of vomeronasal receptor genes, and increased apoptosis and defective differentiation of VSNs. Interestingly, the loss of Bcl11b function affects the fate choice between the two types of VSNs, $G \alpha_{i 2}$ - and $G \alpha_{o}$-positive neurons, in opposite ways. Together, these data indicate that $\mathrm{Bcl11b}$ plays an essential role in the development of the vomeronasal system and in the regulation of the fate choice of VSNs.

\section{Bcl11b is required for the survival and differentiation of vomeronasal sensory neurons}

The molecular mechanisms that control the developmental processes of the VSN have not been well characterized. Genetic studies by Murray et al. (2003) have demonstrated that Mash1 is a determining factor for the neuronal lineage of VSN in neurogenesis and suggested that the molecular details of neurogenesis in the $\mathrm{VNE}$ and MOE are similar. In the MOE, the Mash $1>\mathrm{Ngn} 1>$ NeuroD genetic pathway is involved in neuronal differentiation (Cau et al., 2002). We showed that these bHLH transcription factors were expressed in the VNE, and the expression patterns were similar to the expression patterns in the MOE. These results suggest that the genetic pathway is conserved during the neuronal differentiation of VSNs because it is comparable to the pathway in 
A
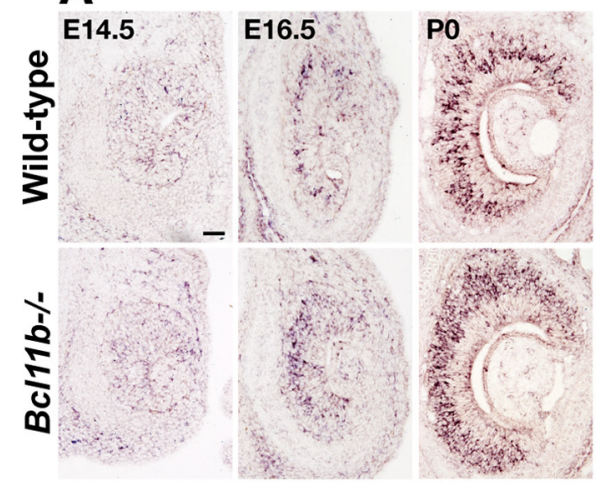

B
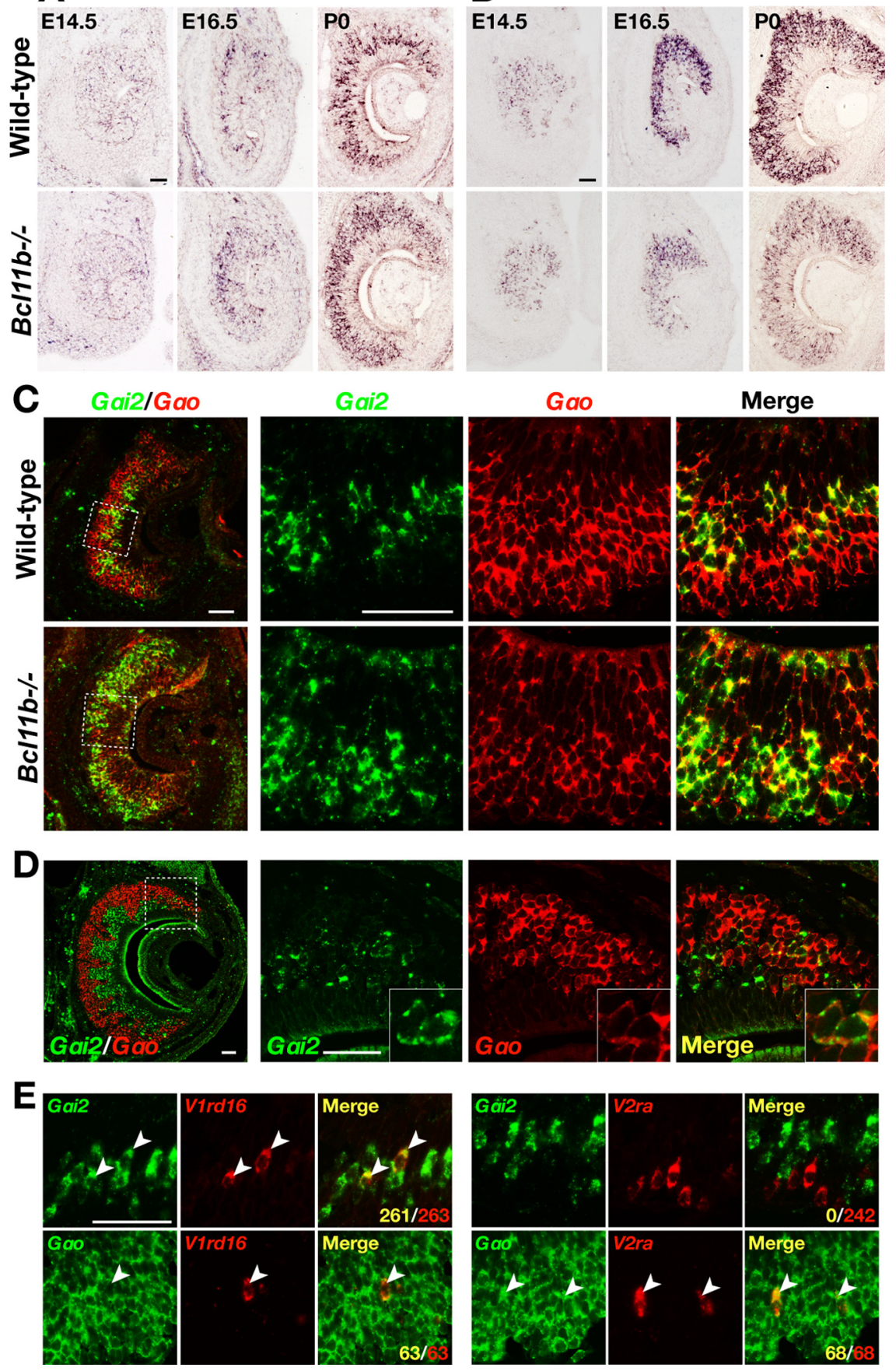

Figure 9. Abnormal development and localization of the two types of VSNs in the VNE of $B C 111 b^{-1-}$ mice. $A$, ISH with a $G \alpha_{i 2}$ probe in coronal sections of the $B d 111 b^{-/-}$and wild-type VNE at E14.5, E16.5, and PO. At E14.5, the expression of $G \alpha_{i 2}$ was rarely observed in either $B C l 11 b^{-/-}$or wild-type embryos. At E16.5 and P0, $G \alpha_{i 2}$-expressing cells were increased and were distributed more broadly in the apical- basal axis in the VNE of $B d 111 b^{-/-}$mice compared with wild-type mice. $B$, ISH using the $G \alpha_{o}$ probe in coronal sections of the $B C / 11 b^{-\prime-}$ and wild-type VNE at E14.5, E16.5, and P0. AtE14.5, expression of $G \alpha_{o}$ was detected in the VNE of $B C 111 b^{-/-}$mice and in wild-type embryos. At E16.5 and P0, the expression of $G \alpha_{o}$ was decreased in both number and level in the $B d 111 b^{-/-}$VNE. C, Two-color ISH using the $G \alpha_{i 2}$ probe (green) and the $G \alpha_{o}$ (red) probe in coronal sections of the $B d 111 b^{-/-}$ and wild-type VNE at P0. Most $G \alpha_{i 2}$-positive cells were $G \alpha_{0}$ positive in both $B C 111 b^{-1-}$ and wild-type mice. $G \alpha_{i 2} / G \alpha_{o}$ doublepositive cells were increased in the VNE of $B C 111 b^{-1-}$ mice, but $G \alpha_{o}$ single-positive cells were decreased. $\boldsymbol{D}$, Coexpression of $G \alpha_{i 2}$ and $G \alpha_{o}$ were analyzed by two-color ISH in the adult VNE (left: low magnification; right: high magnification of the dotted box). Coexpression of $G \alpha_{i 2}$ (green) and $G \alpha_{o}$ (red) were observed in VSNs of the marginal region (typical coexpressing cells are shown in the inset). $\boldsymbol{E}$, Coexpression of $V 1 r d 16$ (red) with either $G \alpha_{i 2}$ or $G \alpha_{o}$ (green) and coexpression of $V 2 r a$ (red) with either $G \alpha_{i 2}$ or $G \alpha_{o}$ (green) in the wild-type VNE were analyzed using two-color ISH at P0. V1rd16-positive VSNs coexpressed both $G \alpha_{i 2}$ and $G \alpha_{o}$ (arrowheads). However, V2ra-positive VSNs coexpressed $G \alpha_{o}$ (arrowheads), but not $G \alpha_{i 2}$. Scale bars: $50 \mu \mathrm{m}$. the OSN that was previously proposed (Murray et al., 2003; Suzuki et al., 2003). This study adds Bcl1 $1 \mathrm{~b}$ as one of the important transcription factors that regulate the differentiation of VSNs.

During the process of differentiation of VSNs, Bcl11b is highly expressed in SCG10-positive postmitotic immature neurons and is decreased in mature neurons. This stage-specific expression of $B c l 11 b$ during neuronal differentiation is similar to that in the neocortex and the striatum, where $B c l 11 b$ is expressed at high levels in postmitotic neurons but not in progenitor cells (Arlotta et al., 2005, 2008) and plays important roles in postmitotic mature differentiation. In the $B c l 11 b^{-/-} \mathrm{VNE}$, the correct number of neurons is produced, but most of these neurons fail to differentiate to maturity, which leads to increased apoptosis in GAP43-positive immature neurons. Therefore, the observed abnormalities in VSN differentiation were most likely caused by defects in postmitotic maturation rather than by an earlier defect in precursor cells. These results indicate that $\mathrm{Bcl} 11 \mathrm{~b}$ is required for the survival and mature differentiation of postmitotic VSNs.

The molecular mechanisms that underlie the increased apoptosis, impaired axonal projections and defective mature differentiation of VSNs in $\mathrm{Bcl1} 1 b^{-/-}$mice remain unclear. Because $B c l 11 b$ is expressed in both VSNs and their axonal target, the AOB, the phenotypes observed in VSNs could be due to a cell-autonomous effect of the loss of Bcl11b function, a non-cell-autonomous effect, or both. In non-cell-autonomous mechanisms, the $B c l 11 b^{-/-}$AOB may somehow influence the mature differentiation of VSNs and/or cause apoptosis. To reveal this, it will be interesting to perform rescue experiments by forced expression of $\mathrm{Bcl1} 1 \mathrm{~b}$ specifically in either VSNs or in mitral/tufted cells in the AOB. Nevertheless, we speculate that a non-cell-autonomous effect is unlikely due to the following reasons: (1) there are no obvious defects observed in cellular composition and survival in the AOB, except for the lack of the vomeronasal nerve layer; and (2) in the closely related main olfactory system, genetic or surgical removal of the OB, which is the axonal target of olfactory sensory neurons, does not significantly influence cellular proliferation, the differentiation of the olfactory epithelium, or odorant receptor gene expression (Graziadei et al., 1978; Sullivan et al., 1995; St John et al., 2003).

In the immune system, the germline deletion of $B c l 11 b$ results in impaired T-cell development around CD4/CD8 double- 
negative stages 2 and 3 and defective T-cell receptor gene expression due to unsuccessful recombination, which consequently causes apoptosis (Wakabayashi et al., 2003b; Ikawa et al., 2010; Li et al., 2010a,b). As designed for the detection of foreign molecules, the vomeronasal system shares similarities with the immune system. Both systems are responsible for self- vs nonself-recognition by expressing one receptor per cell from a large repertoire of receptor genes in a monoallelic and mutually exclusive manner. It is conceivable that $\mathrm{Bcl11b}$ may regulate this common and unique mode of gene expression. In future experiments, it will be interesting to investigate genomic region tethering of the transcription factor, Bcl11b, using chromatin immunoprecipitation (ChIP) on DNA chip analysis and ChIP sequencing analysis in combination with microarray data to understand the molecular mechanism that underlies the Bcl11b-mediated regulation of VSN development.

\section{Bcl11b regulates the fate choice between two types of vomeronasal sensory neurons}

VSNs can be classified into two major types of neurons: $\mathrm{V} 1 \mathrm{r} / \mathrm{G} \alpha_{\mathrm{i} 2}{ }^{-}$ positive and $\mathrm{V} 2 \mathrm{r} / \mathrm{G} \alpha_{\mathrm{o}}$-positive. The fact that both types of VSNs are produced by progenitors in a Mash1-dependent manner and develop side by side in the VNE has raised the question of when their fates are determined and how this dichotomy is regulated. A recent study that used BrdU pulse-labeling experiments suggested that $G \alpha_{i 2}$ - and $G \alpha_{o}$-positive neurons mature independently of each other (de la Rosa-Prieto et al., 2010). Our observations provide the different and novel view that the dichotomy of VSNs is regulated by Bcl11b postmitotically. First, we found that all $G \alpha_{i 2}$-expressing neurons coexpressed $G \alpha_{o}$ in the embryonic VNE (Fig. 9C), and that this coexpression was observed in the marginal region of the adult VNE, an area where newly differentiated and postmitotic immature neurons are located. Second, $G \alpha_{i 2} / G \alpha_{o}$ double-positive VSNs expressed $V 1 r$, but not $V 2 r$, at $\mathrm{P} 0$, indicating commitment to the $\mathrm{V} 1 \mathrm{r} / \mathrm{G} \alpha_{\mathrm{i} 2}$ VSN lineage. These results suggest that $\mathrm{V} 1 \mathrm{r} / \mathrm{G} \alpha_{\mathrm{i} 2}$-type VSNs are generated via $G \alpha_{i 2} / G \alpha_{o}$ double-positive neurons. Third, a small number of $G \alpha_{o}$-expressing cells colabeled with the differentiating/postmitotic neuron marker NeuroD, but none of the $G \alpha_{i 2}$-expressing cells were NeuroD-positive in the VNE (data not shown), indicating that the expression of $G \alpha_{o}$ is earlier than $G \alpha_{i 2}$ in the course of the differentiation of VSNs. Fourth, we detected the expression of $G \alpha_{o}$ at E14.5. However, the expression of $G \alpha_{i 2}$ was observed later, around E16.5, which indicates that $G \alpha_{o}$ single-positive cells exist solely in the early development of the VNE. Therefore, it is likely that the early postmitotic neuron first expresses $G \alpha_{o}$, and then the $G \alpha_{i 2} / G \alpha_{o^{-}}$ positive cell is generated for the $\mathrm{V} 1 \mathrm{r} / \mathrm{G} \alpha_{\mathrm{i} 2} \mathrm{VSN}$ lineage. It is conceivable that $G \alpha_{o}$-positive neurons may be the default type of VSNs. It is interesting that phylogenetically ancient animals, such as fish and frogs, have larger numbers of $V 2 r$ genes than $V 1 r$ genes (Shi and Zhang, 2007), which suggests that they predominantly use and develop V2r/G $\alpha_{\mathrm{o}}$ VSNs.

In addition, we demonstrated that the loss of Bcl11b function affects the number of two types of VSNs in opposite ways (i.e., the number of $G \alpha_{i 2} / G \alpha_{o}$ double-positive apical VSNs increases, but the number of $G \alpha_{o}$ single-positive basal VSNs decreases). This phenotype is supported by the additional observation of the expression of Meis 2 and Tcfap2e, which are novel identifiers of apical and basal

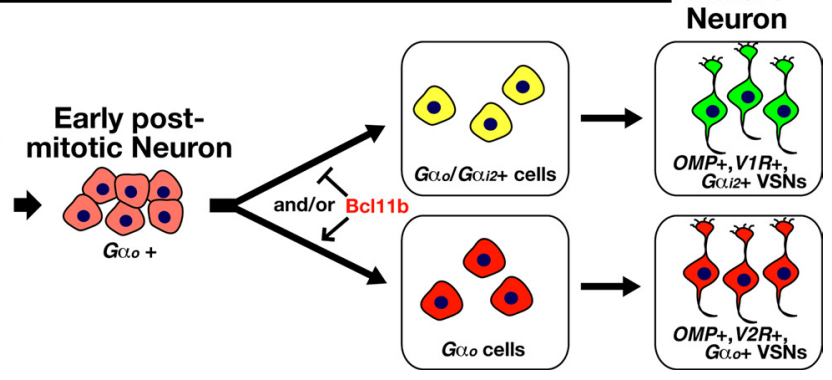

Goo+ VSiNs

Figure 10. A model of the fate determination of vomeronasal sensory neurons. Mash1 is required for the generation of both es of VSNs and acts upstream of $\mathrm{Ngn} 1$ and $B \mathrm{C} / 11 \mathrm{~b}$. Two types of neurons are segregated from each other and from the common VSN lineage. However, the loss of Bd11b function disturbs the fate determination balance between the two types of VSNs, which ositive neurons and decreased $\mathrm{V} 2 \mathrm{r} / \mathrm{G} \alpha_{0}$-positive neurons.

VSNs, respectively. Because the number of cells in the VNE was unaltered in $B c l 11 b^{-1-}$ mice, it is likely that the balance of the fate choice between the V1r/G $\alpha_{\mathrm{i} 2}$ and $\mathrm{V} 2 \mathrm{r} / \mathrm{G} \alpha_{\mathrm{o}} \mathrm{VSN}$ s was disturbed by the loss of Bcl11b activity. These results led us to propose a model in which the dichotomy of VSNs is regulated by Bcl11b (Fig. 10). In this model, Bcl11b regulates the fate choice in early postmitotic neurons to determine the type of VSN, either $G \alpha_{i 2}$-positive or $G \alpha_{o}$-positive, by suppressing the $G \alpha_{i 2}$-positive pathway and/or activating the pathway for the $G \alpha_{o}$-positive VSNs. The role of Bcl11b in fate determination has been demonstrated in other systems. In the immune system, Bcl11b is required for early T-cell lineage commitment by the suppression of other pathways for the determination of the fates of natural killer, myeloid, and dendritic cells (Wakabayashi et al., 2003a; Ikawa et al., 2010; Li et al., 2010a,b). In the CNS, Bcl11b is involved in the fate choice of subcortical projection neurons (Chen et al., 2008) and in the pathways of medium spiny neuron specification and differentiation (Arlotta et al., 2008), which suggests a common function of Bcl1 $1 \mathrm{~b}$ in fate choice during differentiation in different systems by the control of the expression of downstream genes.

\section{Notes}

Supplemental material for this article is available at http://www.hirota.bio. titech.ac.jp/publication/JNeuroscience_2011Bcl11b.pdf. Additional images of Bcl1 $1 \mathrm{~b}$ expression analysis and coexpression analysis of NeuroD with $\mathrm{G} \alpha \mathrm{O}$ and $\mathrm{G} \alpha \mathrm{i} 2$ are presented. This material has not been peer reviewed.

\section{References}

Albu DI, Feng D, Bhattacharya D, Jenkins NA, Copeland NG, Liu P, Avram D (2007) BCL11B is required for positive selection and survival of doublepositive thymocytes. J Exp Med 204:3003-3015.

Arlotta P, Molyneaux BJ, Chen J, Inoue J, Kominami R, Macklis JD (2005) Neuronal subtype-specific genes that control corticospinal motor neuron development in vivo. Neuron 45:207-221.

Arlotta P, Molyneaux BJ, Jabaudon D, Yoshida Y, Macklis JD (2008) Ctip2 controls the differentiation of medium spiny neurons and the establishment of the cellular architecture of the striatum. J Neurosci 28:622-632.

Avram D, Fields A, Pretty On Top K, Nevrivy DJ, Ishmael JE, Leid M (2000) Isolation of a novel family of $\mathrm{C}(2) \mathrm{H}(2)$ zinc finger proteins implicated in transcriptional repression mediated by chicken ovalbumin upstream promoter transcription factor (COUP-TF) orphan nuclear receptors. J Biol Chem 275:10315-10322.

Berghard A, Buck LB (1996) Sensory transduction in vomeronasal neurons: evidence for $\mathrm{G} \alpha \mathrm{o}, \mathrm{G} \alpha \mathrm{i} 2$, and adenylyl cyclase II as major components of a pheromone signaling cascade. J Neurosci 16:909-918.

Brennan PA, Zufall F (2006) Pheromonal communication in vertebrates. Nature 444:308-315.

Camoletto P, Colesanti A, Ozon S, Sobel A, Fasolo A (2001) Expression of 
stathmin and SCG10 proteins in the olfactory neurogenesis during development and after lesion in the adulthood. Brain Res Bull 54:19-28.

Cau E, Gradwohl G, Fode C, Guillemot F (1997) Mash1 activates a cascade of bHLH regulators in olfactory neuron progenitors. Development 124:1611-1621.

Cau E, Casarosa S, Guillemot F (2002) Mash1 and Ngn1 control distinct steps of determination and differentiation in the olfactory sensory neuron lineage. Development 129:1871-1880.

Chen B, Wang SS, Hattox AM, Rayburn H, Nelson SB, McConnell SK (2008) The Fezf2-Ctip2 genetic pathway regulates the fate choice of subcortical projection neurons in the developing cerebral cortex. Proc Natl Acad Sci U S A 105:11382-11387.

Cherrier T, Suzanne S, Redel L, Calao M, Marban C, Samah B, Mukerjee R, Schwartz C, Gras G, Sawaya BE, Zeichner SL, Aunis D, Van Lint C, Rohr O (2009) p21(WAF1) gene promoter is epigenetically silenced by CTIP2 and SUV39H1. Oncogene 28:3380-3389.

Cismasiu VB, Paskaleva E, Suman Daya S, Canki M, Duus K, Avram D (2008) BCL11B is a general transcriptional repressor of the HIV-1 long terminal repeat in Tlymphocytes through recruitment of the NuRD complex. Virology 380:173-181.

Cuschieri A, Bannister LH (1975) The development of the olfactory mucosa in the mouse: light microscopy. J Anat 119:277-286.

de la Rosa-Prieto C, Saiz-Sanchez D, Ubeda-Bañon I, Argandoña-Palacios L, Garcia-Muñozguren S, Martinez-Marcos A (2010) Neurogenesis in subclasses of vomeronasal sensory neurons in adult mice. Dev Neurobiol 70:961-970

Duggan CD, DeMaria S, Baudhuin A, Stafford D, Ngai J (2008) Foxg1 is required for development of the vertebrate olfactory system. J Neurosci 28:5229-5239.

Dulac C, Axel R (1995) A novel family of genes encoding putative pheromone receptors in mammals. Cell 83:195-206.

Dulac C, Torello AT (2003) Molecular detection of pheromone signals in mammals: from genes to behaviour. Nat Rev Neurosci 4:551-562.

Faedo A, Ficara F, Ghiani M, Aiuti A, Rubenstein JL, Bulfone A (2002) Developmental expression of the T-box transcription factor T-bet/Tbx21 during mouse embryogenesis. Mech Dev 116:157-160.

Garrosa M, Gayoso MJ, Esteban FJ (1998) Prenatal development of the mammalian vomeronasal organ. Microsc Res Tech 41:456-470.

Golonzhka O, Metzger D, Bornert JM, Bay BK, Gross MK, Kioussi C, Leid M (2009a) Ctip2/Bcl11b controls ameloblast formation during mammalian odontogenesis. Proc Natl Acad Sci U S A 106:4278-4283.

Golonzhka O, Liang X, Messaddeq N, Bornert JM, Campbell AL, Metzger D, Chambon P, Ganguli-Indra G, Leid M, Indra AK (2009b) Dual role of COUP-TF-interacting protein 2 in epidermal homeostasis and permeability barrier formation. J Invest Dermatol 129:1459-1470.

Graziadei PP, Levine RR, Graziadei GA (1978) Regeneration of olfactory axons and synapse formation in the forebrain after bulbectomy in neonatal mice. Proc Natl Acad Sci U S A 75:5230-5234.

Guillemot F, Lo LC, Johnson JE, Auerbach A, Anderson DJ, Joyner AL (1993) Mammalian achaete-scute homolog 1 is required for the early development of olfactory and autonomic neurons. Cell 75:463-476.

Halpern M, Martínez-Marcos A (2003) Structure and function of the vomeronasal system: an update. Prog Neurobiol 70:245-318.

Herrada G, Dulac C (1997) A novel family of putative pheromone receptors in mammals with a topographically organized and sexually dimorphic distribution. Cell 90:763-773.

Hirota J, Mombaerts P (2004) The LIM-homeodomain protein Lhx2 is required for complete development of mouse olfactory sensory neurons. Proc Natl Acad Sci U S A 101:8751-8755.

Hirota J, Omura M, Mombaerts P (2007) Differential impact of Lhx2 deficiency on expression of class I and class II odorant receptor genes in mouse. Mol Cell Neurosci 34:679-688.

Ikawa T, Hirose S, Masuda K, Kakugawa K, Satoh R, Shibano-Satoh A, Kominami R, Katsura Y, Kawamoto H (2010) An essential developmental checkpoint for production of the T cell lineage. Science 329:93-96.

Ikeda K, Ookawara S, Sato S, Ando Z, Kageyama R, Kawakami K (2007) Six1 is essential for early neurogenesis in the development of olfactory epithelium. Dev Biol 311:53-68.

Ishii T, Hirota J, Mombaerts P (2003) Combinatorial coexpression of neural and immune multigene families in mouse vomeronasal sensory neurons. Curr Biol 13:394-400.

Ishii T, Omura M, Mombaerts P (2004) Protocols for two- and three-color fluorescent RNA in situ hybridization of the main and accessory olfactory epithelia in mouse. J Neurocytol 33:657-669.

Jia C, Halpern M (1996) Subclasses of vomeronasal receptor neurons: differential expression of $\mathrm{G}$ proteins (Gi alpha 2 and $\mathrm{G}(\mathrm{o}$ alpha)) and segregated projections to the accessory olfactory bulb. Brain Res 719:117-128.

Kaneko-Goto T, Yoshihara S, Miyazaki H, Yoshihara Y (2008) BIG-2 mediates olfactory axon convergence to target glomeruli. Neuron 57:834-846.

Kawauchi S, Kim J, Santos R, Wu HH, Lander AD, Calof AL (2009) Foxg1 promotes olfactory neurogenesis by antagonizing Gdf11. Development 136:1453-1464.

Keverne EB (1999) The vomeronasal organ. Science 286:716-720.

Leid M, Ishmael JE, Avram D, Shepherd D, Fraulob V, Dollé P (2004) CTIP1 and CTIP2 are differentially expressed during mouse embryogenesis. Gene Expr Patterns 4:733-739.

Li L, Leid M, Rothenberg EV (2010a) An early T cell lineage commitment checkpoint dependent on the transcription factor Bcl11b. Science 329:89-93.

Li P, Burke S, Wang J, Chen X, Ortiz M, Lee SC, Lu D, Campos L, Goulding D, Ng BL, Dougan G, Huntly B, Gottgens B, Jenkins NA, Copeland NG, Colucci F, Liu P (2010b) Reprogramming of T cells to natural killer-like cells upon Bcl11b deletion. Science 329:85-89.

Marban C, Suzanne S, Dequiedt F, de Walque S, Redel L, Van Lint C, Aunis D, Rohr O (2007) Recruitment of chromatin-modifying enzymes by CTIP2 promotes HIV-1 transcriptional silencing. EMBO J 26:412-423.

Matsunami H, Buck LB (1997) A multigene family encoding a diverse array of putative pheromone receptors in mammals. Cell 90:775-784.

Murray RC, Navi D, Fesenko J, Lander AD, Calof AL (2003) Widespread defects in the primary olfactory pathway caused by loss of Mash1 function. J Neurosci 23:1769-1780.

Rodriguez I, Del Punta K, Rothman A, Ishii T, Mombaerts P (2002) Multiple new and isolated families within the mouse superfamily of V1r vomeronasal receptors. Nat Neurosci 5:134-140.

Ryba NJ, Tirindelli R (1997) A new multigene family of putative pheromone receptors. Neuron 19:371-379.

Schlüter C, Duchrow M, Wohlenberg C, Becker MH, Key G, Flad HD, Gerdes J (1993) The cell proliferation-associated antigen of antibody Ki-67: a very large, ubiquitous nuclear protein with numerous repeated elements, representing a new kind of cell cycle-maintaining proteins. J Cell Biol 123:513-522.

Senawong T, Peterson VJ, Avram D, Shepherd DM, Frye RA, Minucci S, Leid M (2003) Involvement of the histone deacetylase SIRT1 in chicken ovalbumin upstream promoter transcription factor (COUP-TF)-interacting protein 2-mediated transcriptional repression. J Biol Chem 278:43041-43050.

Shi P, Zhang J (2007) Comparative genomic analysis identifies an evolutionary shift of vomeronasal receptor gene repertoires in the vertebrate transition from water to land. Genome Res 17:166-174.

St John JA, Clarris HJ, McKeown S, Royal S, Key B (2003) Sorting and convergence of primary olfactory axons are independent of the olfactory bulb. J Comp Neurol 464:131-140.

Sullivan SL, Bohm S, Ressler KJ, Horowitz LF, Buck LB (1995) Targetindependent pattern specification in the olfactory epithelium. Neuron 15:779-789.

Suzuki Y, Mizoguchi I, Nishiyama H, Takeda M, Obara N (2003) Expression of Hes6 and NeuroD in the olfactory epithelium, vomeronasal organ and non-sensory patches. Chem Senses 28:197-205.

Takami S, Fernandez GD, Graziadei PP (1992) The morphology of GABAimmunoreactive neurons in the accessory olfactory bulb of rats. Brain Res 588:317-323.

Topark-Ngarm A, Golonzhka O, Peterson VJ, Barrett B Jr, Martinez B, Crofoot K, Filtz TM, Leid M (2006) CTIP2 associates with the NuRD complex on the promoter of p57KIP2, a newly identified CTIP2 target gene. J Biol Chem 281:32272-32283.

Wakabayashi Y, Inoue J, Takahashi Y, Matsuki A, Kosugi-Okano H, Shinbo T, Mishima Y, Niwa O, Kominami R (2003a) Homozygous deletions and point mutations of the Rit1/Bcl11b gene in gamma-ray induced mouse thymic lymphomas. Biochem Biophys Res Commun 301:598-603.

Wakabayashi Y, Watanabe H, Inoue J, Takeda N, Sakata J, Mishima Y, Hitomi J, Yamamoto T, Utsuyama M, Niwa O, Aizawa S, Kominami R (2003b) $\mathrm{Bcl1} 1 \mathrm{~b}$ is required for differentiation and survival of alphabeta T lymphocytes. Nat Immunol 4:533-539.

Yoshihara S, Omichi K, Yanazawa M, Kitamura K, Yoshihara Y (2005) Arx homeobox gene is essential for development of mouse olfactory system. Development 132:751-762. 